\title{
Wireless Systems and Interference Avoidance
}

\author{
Christopher Rose, Member, IEEE, Sennur Ulukus, Member, IEEE, and Roy D. Yates, Member, IEEE
}

\begin{abstract}
Motivated by the emergence of programmable radios, we seek to understand a new class of communication system where pairs of transmitters and receivers can adapt their modulation/demodulation method in the presence of interference to achieve better performance. Using signal to interference ratio as a metric and a general signal space approach, we present a class of iterative distributed algorithms for synchronous systems which results in an ensemble of optimal waveforms for multiple users connected to a common receiver (or colocated independent receivers). That is, the waveform ensemble meets the Welch Bound with equality and, therefore, achieves minimum average interference over the ensemble of signature waveforms. We derive fixed points for a number of scenarios, provide examples, look briefly at ensemble stability under user addition and deletion as well as provide a simplistic comparison to synchronous code-division multiple-access. We close with suggestions for future work.
\end{abstract}

Index Terms-Adaptive modulation, code-division multiple-access systems, codeword optimization, interference avoidance, multiuser detection, sum capacity, vector channels.

\section{INTRODUCTION}

W IRELESS SYSTEM designers have always had to contend with interference from both natural sources and other users of the medium. Thus, the classical wireless communications design cycle has consisted of measuring or predicting channel impairments, choosing a modulation method, signal preconditioning at the transmitter, and processing at the receiver to reliably reconstruct the transmitted information. These methods have evolved from simple (like FM and preemphasis) to relatively complex (like code-division multiple-access (CDMA) and adaptive equalization). However, all share a common attribute-once the modulation method is chosen, it is difficult to change. For example, an amplitude shift-keying (ASK) system cannot be simply modified to obtain a phase shift-keying (PSK) system owing to the complexities of the transmission and reception hardware. Universal radios ${ }^{1}$ [1]-[5] change this paradigm by providing the communications engineer with a radio which can be programmed to produce almost arbitrary output waveforms and act as an almost arbitrary receiver type. Thus, it is no longer unthinkable to instruct

Manuscript received May 18, 2000; revised August 21, 2001; accepted August 28,2001 . The editor coordinating the review of this paper and approving it for publication is S. Tekinay. This work was supported in part by National Science Foundation under Grant CCR-99-73012.

C. Rose and R. D. Yates are with WINLAB, Department of Electrical and Computer Engineering, Rutgers University, Piscataway, NJ 08854-8060 USA (e-mail: crose@winlab.rutgers.edu; ryates@winlab.rutgers.edu

S. Ulukus was with WINLAB, Department of Electrical and Computer Engineering, Rutgers University, Piscataway, NJ 08854-8060 USA. She is now with the Department of Electrical and Computer Engineering, University of Maryland, College Park, MD 20742 USA (e-mail: ulukus@umd.eng.edu).

Publisher Item Identifier 10.1109/TWC.2002.800540.

${ }^{1}$ Also often called software radio. the transmitting and receiving radios to use a more effective modulation in a given situation. Of course, practical radios of this sort are probably many years away. Nonetheless, if Moore's law holds true, they are certainly on the not-too-distant horizon.

It is, therefore, probable that wireless systems of the near future will have elements which adapt dynamically to changing patterns of interference by adjusting modulation and processing methods in much the same way that power control [6]-[10], is used today, albeit on a possibly slower time scale. Furthermore, if the release of $300 \mathrm{MHz}$ of unlicensed spectrum in the $5-\mathrm{GHz}$ range [11] is any indication, one might expect there to be an abundance of mutually interfering independent systems and no central control for efficient coordination. This provides added impetus to understand mutual interference of systems at some general level and implicit coordination in a multisystem environment.

In this paper, we consider radios which can vary their output waveforms as well as their demodulation method. Our development starts with signal space to drive home the point that these techniques are applicable to a broad variety of communications scenarios and not only the usual "chip-based" CDMA system with a nondispersive channel and a single antenna. That is, if the problem has a signal space description, then interference avoidance can be brought to bear. ${ }^{2}$

In this context, we consider optimal waveform selection to maximize the signal-to-interference ratio (SIR) for a power-constrained user in the presence of interference. Starting from the essentially classical approach of whitening [12] and showing the relation to modern methods (typified by [13]), we then consider ensembles of users and describe a class of distributed greedy algorithm which can optimize their shared use of the medium. That is, through local self-interested action, a social optimum can usually be reached. Moreover, the algorithms we will describe are simple and amenable to adaptive implementation.

\section{BACKGROUND}

Consider the classical continuous time digital communications model, in which during an interval $[0, T]$, a signal $b \sqrt{p} S(t)$ is transmitted where $b= \pm 1$ equiprobably, $p$ is the received power, and $S(t)$ is the (unit energy/power) signal waveform. A receiver recovers $r(t)=b \sqrt{p} S(t)+Z(t)$, where $Z(t)$ is an independent interference stochastic waveform that may be composed of both thermal noise and interfering signals of other transmitters. For a single bit, the fundamental problem is to

\footnotetext{
${ }^{2}$ Section II provides a self-contained development of the basic ideas for students, those new to the field, and those who like to reread good stories. It also shows how interference avoidance stems naturally from much older work (whitening filters) though under a new guise (linear receivers for multiuser detection). The knowledgeable reader can safely skim Section II with no loss of continuity.
} 
build a receiver which guesses $b$ with minimum probability of error. Alternatively, when $b$ is one bit in a stream of coded bits, we would like to produce a soft estimate of $b$ with high signal to interference ratio (SIR). When $Z(t)$ is composed of known waveforms in addition to independent Gaussian noise, that is $Z(t)=\sum_{i} b_{i} \sqrt{p_{i}} S_{i}(t)+N(t)$, multiuser receivers have been designed for a variety of objectives, e.g., minimum probability of error, maximum SIR, or zero interference from other users [13]. These multiuser systems share the property that the receiver does as best it can given the set of transmitter signals $S_{i}(t)$.

From the perspective of the receiver for $S(t)$, the interference $Z(t)$ is simply a stochastic process, which we assume zero-mean with no loss of generality. Ideally, we would like to obtain a set of uncorrelated (and preferably independent) sufficient statistics and then optimally combine these either to detect the bit $b$ or to derive an estimate of $b$. When $Z(t)$ is Gaussian, these projections would indeed be independent Gaussian random variables and the optimal detection problem would be easily solved. A complete and rigorous development of the ideas can be found in [12]. Here, we provide a brief recapitulation.

In general, given a stochastic process, $Z(t)$ we seek an orthonormal representation

$$
Z(t)=\lim _{N \rightarrow \infty} \sum_{i=1}^{N} a_{i} \Phi_{i}(t)
$$

with $a_{i}=\int_{0}^{T} Z(t) \Phi_{i}(t) d t$. Note that in (1), the convergence requirement is not the usual pointwise limit, but the limit in the mean criterion. The interested reader is referred to [12] and [14] for further details. For our purposes, we assume that such an expansion for $Z(t)$ exists and converges. Now, we seek a special set of orthonormal $\Phi_{i}$ which produce uncorrelated projections.

$$
\begin{aligned}
E\left[\left\langle\Phi_{i}(t), Z(t)\right\rangle\left\langle\Phi_{j}(t), Z(t)\right\rangle\right] & = \\
E\left[\int_{0}^{T} \int_{0}^{T} \Phi_{i}(\tau) Z(\tau) \Phi_{j}(t) Z(t) d t d \tau\right] & =\lambda_{j} \delta_{i j} .
\end{aligned}
$$

Propagating the expectation and defining $R_{Z}(t, \tau)=$ $E[Z(t) Z(\tau)]$, we obtain the integral equation

$$
\int_{0}^{T} \Phi_{j}(t)\left(\int_{0}^{T} R_{Z}(t, \tau) \Phi_{i}(\tau) d \tau\right) d t=\lambda_{j} \delta_{i j}
$$

The solution to this integral equation requires

$$
\lambda_{i} \Phi_{i}(t)=\int_{0}^{T} R_{Z}(t, \tau) \Phi_{i}(\tau) d \tau .
$$

For the interested reader, the properties of (4) are discussed at some length in [12].

Since integral equations are in general difficult to solve, it is useful to derive an equivalent discrete representation of (4). This will allow us to use simple methods from linear algebra. Now let us assume that $Z(t)$ and, therefore, the function set $\left\{\Phi_{i}(t)\right\}$, can be well-approximated by a finite set of orthonormal basis functions $\left\{\Psi_{n}(t)\right\}$ on the interval $[0, T]$. That is, we assume that the process $Z(t)$ has no significant energy outside some finite signal space. As an example, a process "almost" limited to bandwidth $\pm W$ has a basis function set with about $2 W T$ orthonormal functions [12], [15], [16]. Likewise, for a synchronous CDMA system with $N$ chips per bit, the appropriate orthonormal set consists of the $N$ time-shifted chip pulses. One could also use a space-time orthogonalization for reception/transmission antenna diversity and/or a frequency-time orthogonalization for a frequency-hopped system.

Regardless of the specifics, once we assume a convenient finite basis function set over the interval, the $\Phi_{i}(t)$ can then be represented by the finite sum

$$
\Phi_{i}(t)=\sum_{n=1}^{N} \phi_{\mathrm{in}} \Psi_{n}(t) .
$$

Combining (4) and (5) yields

$$
\lambda_{i} \sum_{n=1}^{N} \phi_{\mathrm{in}} \Psi_{n}(t)=\int_{0}^{T} R_{Z}(t, \tau) \sum_{n=1}^{N} \phi_{\mathbf{i n}} \Psi_{n}(\tau) d \tau .
$$

Further, projecting the right and left hand sides onto $\Psi_{k}(t)$ yields

$$
\begin{aligned}
\lambda_{i} \phi_{i k} & =\sum_{n=1}^{N} \phi_{\text {in }} \underbrace{\int_{0}^{T} \int_{0}^{T} R_{Z}(t, \tau) \Psi_{k}(t) \Psi_{n}(\tau) d t d \tau}_{r_{k n}} \\
& =\sum_{n=1}^{N} r_{k n} \phi_{\text {in }} .
\end{aligned}
$$

We may also rewrite the $r_{n k}$ directly in terms of the projections $Z_{n}=\int_{0}^{T} Z(t) \Phi_{n}(t) d t$ as

$$
r_{n k}=E\left[\int_{0}^{T} \int_{0}^{T} Z(t) Z(\tau) \Psi_{k}(t) \Psi_{n}(\tau) d t d \tau\right]=E\left[Z_{k} Z_{n}\right] .
$$

Thus, (7) is a reduction of the continuous time integral (4) to a standard matrix eigenvalue/eigenvector equation of the form

$$
E\left[\mathbf{Z Z}^{\top}\right] \boldsymbol{\phi}_{i}=\mathbf{R} \boldsymbol{\phi}_{i}=\lambda_{i} \boldsymbol{\phi}_{i}
$$

where $\boldsymbol{\phi}_{i}=\left[\phi_{i 1} \cdots \phi_{i N}\right]^{\top}$ and $\mathbf{Z}=\left[Z_{1} \cdots Z_{N}\right]^{\top}$. Each eigenvector corresponds to an eigenfunction of (4) and it is easily verified that each eigenvalue is the amount of interference signal energy carried by that eigenfunction. It is also easy to verify that since $R_{Z}(t, \tau)$ is an autocorrelation function, $\mathbf{R}$ is symmetric and positive semidefinite. This implies that $\mathbf{R}$ has nonnegative eigenvalues and an associated full set of orthonormal eigenvectors which span $\Re^{N}$ [17].

The receiver observes the signal

$$
r(t)=b S(t)+Z(t)
$$

as input on the interval $[0, T]$. Projecting the received signal onto the interference eigenfunctions $\Phi_{1}(t), \ldots, \Phi_{n}(t)$, we obtain the vector output

$$
\overline{\mathbf{r}}=l \overline{\mathbf{s}}+\overline{\mathbf{z}}
$$

where $\overline{\mathbf{s}}$ and $\overline{\mathbf{z}}$ have $n$th components $\bar{s}_{n}=\left\langle S(t), \Phi_{n}(t)\right\rangle, \bar{z}_{n}=$ $\left\langle Z(t), \Phi_{n}(t)\right\rangle$ and the $\bar{z}_{n}$ are mutually uncorrelated. 
If the $\Phi_{n}(t)$ do not span the signal space, then error-free reception is possible by first augmenting the basis set to span the signal space (via Gram-Schmidt [18] for example) and, then, noting that there will be no interference energy projected onto these augmented basis functions which carry nonzero signal energy. Thus, with no loss of generality, we assume that the basis functions $\Phi_{n}(t)$ also span the signal space for $S(t)$, and $b \overline{\mathbf{s}}$ contains all available information about $b S(t)$.

At this point it is instructive to consider the detection of $b$ when $Z(t)$ is a Gaussian interference process. Because we chose the interference eigenfunctions $\left\{\Phi_{1}(t), \ldots, \Phi_{N}(t)\right\}$ to yield uncorrelated interference components $\bar{z}_{n}$, it is straightforward to show that the likelihood ratio test for optimal detection of $b$ becomes [12], [14]

$$
\sum_{n=1}^{N} \frac{\bar{s}_{n} \bar{r}_{n}}{\lambda_{n}} \underset{\text { say } 0}{\gtrless} 0
$$

where the $\left\{\bar{r}_{n}\right\}$ are the projections of $r(t)$ onto the decorrelating basis functions $\Phi_{n}(t)$. This detection method is called a whitening filter since it can be written as

$$
\sum_{n=1}^{N} \frac{\bar{r}_{n}}{\sqrt{\lambda_{n}}} \frac{\bar{s}_{n}}{\sqrt{\lambda_{n}}} \stackrel{\text { say } 1}{\gtrless} \text { say } 0
$$

which is an initial rescaling of the input to make interference components $\left(\left\{\bar{z}_{n}\right\}\right)$, already uncorrelated, have equal energy $\left(\left\{\bar{z}_{n} / \sqrt{\lambda_{n}}\right\}\right)-$ just as would be the case for a white noise process without rescaling. A matched filter on the rescaled signal vector components $\bar{s}_{n} / \sqrt{\lambda_{n}}$ is then performed to complete the detection process.

It is worthwhile to note that in a CDMA system where $Z(t)$ consists of the other users' known signature waveforms and additive white Gaussian noise (AWGN), the vector $\overline{\mathbf{c}}$ with components $\bar{c}_{n}=\bar{s}_{n} / \lambda_{n}$ is a scaled version of the well known minimum mean squared error (MMSE) linear filter [19] and the decision rule (12) is the MMSE multiuser detector. We see that the filter output (and decision statistic)

$$
X=\overline{\mathbf{c}}^{\top} \overline{\mathbf{r}}=\sum_{n} \bar{c}_{n} \bar{r}_{n}=\left(\sum_{n=1}^{N} \frac{\bar{s}_{n}^{2}}{\lambda_{n}}\right) b+\sum_{n=1}^{N} \frac{\bar{s}_{n} \bar{z}_{n}}{\lambda_{n}}
$$

contains both signal and interference terms and that the output SIR is

$$
\begin{aligned}
\operatorname{SIR}_{X}= & \frac{E\left[\left(b \sum_{n=1}^{N} \frac{\bar{s}_{n z}^{2}}{\lambda_{n}}\right)^{2}\right]}{E\left[\left(\sum_{n=1}^{N} \frac{\bar{s}_{n} \bar{z}_{n}}{\lambda_{n}}\right)^{2}\right]} \\
= & \frac{\left(\sum_{n=1}^{N} \frac{\bar{s}_{n z}^{2}}{\lambda_{n}}\right)^{2}}{\sum_{n=1}^{N} \sum_{m=1}^{N} \frac{\bar{s}_{n} \bar{s}_{m} E\left[\bar{z}_{n} \bar{z}_{m}\right]}{\lambda_{n} \lambda_{m}}}=\sum_{n=1}^{N} \frac{\bar{s}_{n}^{2}}{\lambda_{n}}
\end{aligned}
$$

It is well known that among all linear filters $\overline{\mathbf{c}}$, the MMSE filter maximizes the output SIR [19]. However, (15) demonstrates that it remains possible to obtain a higher output SIR by altering the components $\bar{s}_{n}$ of the desired signal $S(t)$. That is, when $S(t)$ is subject to the unit energy constraint $\sum_{n} \bar{s}_{n}^{2}=1$, we can maximize $\operatorname{SIR}_{X}$ by choosing $\bar{s}_{n}=1$ for any $\lambda_{n}=\lambda^{*}=$ $\min _{k} \lambda_{k}$. In this case, we have $S(t)=\Phi_{n}(t)$. Equivalently, we could distribute the signal energy in some arbitrary way over all such $\Phi_{n}(t)$. Regardless, this result has a simple intuitively pleasing physical interpretation: To obtain maximum SIR, place all the signal energy where there is least interference. We call this procedure interference avoidance and for a single user with a given interference process, the method is straightforward. We now examine the implications of this simple Karhunen-Loeve inspired rule for an ensemble of users. We will find that the greedy objective in which a user adapts its signature to improve its SIR has desirable consequences for multiuser systems.

\section{INTERFERENCE AVOIDANCE FOR MULTIPLE USERS}

We now consider a multiuser system in which the received signal $r(t)$ explicitly includes $M$ users and white Gaussian noise. Given the existence of a finite set of $N$ orthonormal basis functions $\Psi_{i}(t)$ for the signal space, we can express the received signal as the vector

$$
\mathbf{r}=\sum_{i=1}^{M} \sqrt{p_{i}} b_{i} \mathbf{s}_{i}+\mathbf{n}
$$

where $\mathbf{n}$ is the projection of the AWGN onto the basis. The classical communications scenario presumes that each user signature $S_{i}(t)$ is fixed. Assuming universal radio receivers and transmitters, we now allow the use of tailored signature waveforms $S_{i}(t)$. Without loss of generality, we assume each $S_{i}(t)$ has unit energy. The relationship between signature selection and multiuser system capacity has been studied in several papers [10], [20], [21]. In [20], it is shown that for a set of users' rates $R_{1}, \ldots, R_{M}$ belonging to the information theoretic achievable rate region $\mathcal{C}$, the sum capacity is

$$
C_{\mathbf{s}}=\max _{\left(R_{1}, \ldots, R_{M}\right) \in \mathcal{C}} \sum_{i=1}^{M} R_{i}=\frac{1}{2} \log \left|\mathbf{I}_{N}+\sigma^{-1} \mathbf{S P S}^{\top}\right|
$$

In (17), $\mathbf{I}_{N}$ is the $N \times N$ identity matrix, $\mathbf{P}$ is the diagonal matrix of user's powers $p_{k}, \mathbf{S}=\left[\mathbf{s}_{1}, \ldots, \mathbf{s}_{M}\right]$ is the $N \times M$ matrix with columns $\mathbf{s}_{i}$ and $\sigma$ is the level of white background noise. Note that these $s_{k}$ are the projections of the $S_{i}(t)$ onto the arbitrary spanning orthogonal basis $\left\{\Psi_{k}(t)\right\}$ so that the $\mathbf{s}_{i}$ may be called generalized codeword vectors and represent arbitrary signals inside an arbitrary (but finite) signal space.

\section{A. Maximizing Symmetric/Sum Capacity}

When the powers of the users are the same, $p_{k}=p$ for all $k$, (17) reduces to

$$
C_{\mathbf{s}}=\frac{1}{2} \log \left|\mathbf{I}_{N}+\frac{p}{\sigma} \mathbf{S S}^{\top}\right|=\frac{1}{2} \log \left|\mathbf{I}_{M}+\frac{p}{\sigma} \mathbf{S}^{\top} \mathbf{S}\right|
$$

where the last equality follows from the fact that for any two matrices $\mathbf{A}_{K \times M}$ and $\mathbf{B}_{M \times K},\left|\mathbf{I}_{K}+\mathbf{A B}\right|=\left|\mathbf{I}_{M}+\mathbf{B A}\right|$. It was shown in [21] that the sum capacity for equal received powers is maximized if the signature sequences are chosen such that if $M \leq N$

$$
\mathbf{S}^{\top} \mathbf{S}=\mathbf{I}_{M}
$$


and if $M \geq N$

$$
\mathbf{S S}^{\top}=\left(\frac{M}{N}\right) \mathbf{I}_{N} .
$$

In [10], the user capacity of a CDMA system is defined in terms of the maximum number of admissible users. Given the signal space dimensionality $N$ and a common SIR target $\beta, M$ users are said to be admissible if there exist positive powers $p_{i}$ and signature sequences $\mathbf{s}_{i}$ such that each user has an SIR at least as large as $\beta$. The user capacity was found for two kinds of linear receiver structures in [10]: matched filters and MMSE filters [19], [22]. It was shown in [10] that the user capacity with MMSE receivers is maximized if the received powers of the users are identical and if the signature sequence set satisfies (19) when $M \leq N$ and (20) when $M \geq N$. When $M \leq N$ and (19) is satisfied or when $M \geq N$ and (20) is satisfied, the MMSE and matched filters are the same. Thus, reference [10] concludes that the user capacity of a system with matched filter receivers is the same as that using MMSE filters.

In [21], the unit energy sequence sets satisfying (20) are called Welch bound equality (WBE) sequence sets. Welch [23] derived the following lower bound on the sum of the squared cross correlations, which we will call total squared correlation (TSC).

$$
\mathrm{TSC}=\operatorname{Trace}\left[\left(\mathbf{S S}^{\top}\right)^{2}\right]=\sum_{i=1}^{M} \sum_{j=1}^{M}\left(\mathbf{s}_{i}^{\top} \mathbf{s}_{j}\right)^{2} \geq \frac{M^{2}}{N} .
$$

For a simple derivation of the bound (21), see [24] and [25]. Note that sequence sets satisfying (20) satisfy the bound (21) with equality. We observe that the set of sequences satisfying either (19) for $M \leq N$ or (20) for $M \geq N$ has the property that the sequence set has minimum TSC. That is, to maximize both sum capacity and user capacity, we should choose sequence sets with minimum TSC.

The relationship between sum capacity and TSC can be made more formal. We start by incorporating the fixed signal power $p_{k}$ into the signal vector energy $\left|\mathbf{s}_{k}\right|^{2}$ for notational clarity. That is, instead of requiring $\left|\mathbf{s}_{k}\right|^{2}=1$, we set $\left|\mathbf{s}_{k}\right|^{2}=p_{k}$. We note that the sum capacity is then

$$
C_{\mathbf{s}}=\frac{1}{2} \log \left|\frac{1}{\sigma}\left(\sigma \mathbf{I}_{N}+\mathbf{S S}^{\top}\right)\right| .
$$

Then, we define the eigenvalues of $\sigma \mathbf{I}_{N}+\mathbf{S S}^{\top}$ as $\lambda_{i}, i=$ $1, \ldots, N$ and rewrite sum capacity as

$$
C_{\mathbf{s}}=-\frac{N}{2} \log \sigma+\frac{1}{2} \sum_{i=1}^{N} \log \lambda_{i}
$$

essentially as was done in both [21] and [26]. Now consider TSC. We first note that if $\left\{\lambda_{i}\right\}$ are the eigenvalues of $\sigma^{2} \mathbf{I}_{N}+$ $\mathbf{S S}^{\top}$ then

$$
\text { Trace }\left[\left(\sigma \mathbf{I}_{N}+\mathbf{S S}^{\top}\right)^{2}\right]=\sum_{i=1}^{N} \lambda_{i}^{2}
$$

since the eigenvalues of $\left(\sigma^{2} \mathbf{I}_{N}+\mathbf{S S}^{\top}\right)^{2}$ must be $\left\{\lambda_{i}^{2}\right\}$ [17].

For brevity's sake, we follow [10], [26] and note that the function described in (23) is Schur concave while that of (24) is Schur convex [27]. Since any constraints on the eigenvalues must be identical, and in fact form a convex set [26], [28], [29], we can conclude that any set $\left\{\lambda_{i}\right\}$ which maximizes (23) must also minimize (24) and vice versa. ${ }^{3}$ Therefore, minimization of TSC is completely equivalent to maximization of $C_{\mathrm{s}}$. Of course it must be emphasized that this result does NOT imply equivalence between TSC and sum or symmetric capacity - but only that minimization of TSC implies maximization of sum capacity.

This result is useful since we will find that TSC minimization is simple and lends itself to a distributed implementation suitable for use in a system of many users.

\section{B. Iterative Methods of TSC Reduction}

There are a number of methods which might be used to determine codeword sets which minimize TSC [10], [21], [26]. Here, we explore simple iterative methods which can be applied by each transmitter/receiver pair asynchronously and independently.

For a single user $k$, we observe that $\mathbf{S S}^{\top}=\mathbf{R}_{k}+\mathbf{s}_{k} \mathbf{S}_{k}^{\top}$ where $\mathbf{R}_{k}=\sum_{i \neq k} \mathbf{s}_{i} \mathbf{s}_{i}^{\top}$, the correlation matrix of the interference faced by user $k$, is analogous to the matrix $\mathbf{R}$ introduced in Section II. For future use, we note that Trace $\left[\mathbf{S S}^{\top}\right]=M$. When user $k$ replaces its signature vector $\mathbf{s}_{k}$ with a vector $\mathbf{x}$, the resulting difference in TSC is

$$
\Delta=\operatorname{Trace}\left[\left(\mathbf{R}_{k}+\mathbf{s}_{k} \mathbf{s}_{k}^{\top}\right)^{2}\right]-\operatorname{Trace}\left[\left(\mathbf{R}_{k}+\mathbf{x x} \mathbf{x}^{\top}\right)^{2}\right] .
$$

After some linear algebraic manipulations, we find that $\Delta \geq 0$ iff

$$
2 \mathbf{s}_{k}^{\top} \mathbf{R}_{k} \mathbf{s}_{k}+\left|\mathbf{s}_{k}\right|^{2} \geq 2 \mathrm{x}^{\top} \mathbf{R}_{k} \mathbf{x}+|\mathbf{x}|^{2}
$$

which reduces to

$$
\mathbf{s}_{k}^{\top} \mathbf{R}_{k} \mathbf{s}_{k} \geq \mathbf{x}^{\top} \mathbf{R}_{k} \mathbf{x}
$$

if $|\mathbf{x}|=\left|\mathbf{s}_{k}\right|$ as we will hereafter assume. When the interference faced by user $k$ includes AWGN with power spectral density $\sigma^{2}$, we may replace $\mathbf{R}_{k}$ by $\mathbf{Z}_{k}=\mathbf{R}_{k}+\sigma^{2} \mathbf{I}$ if desired. As previously, we note that minimizing $\operatorname{Trace}\left[\left(\mathbf{S S}^{\top}\right)^{2}\right]$ is equivalent to minimizing Trace $\left[\left(\mathbf{S S}^{\top}+\sigma^{2} \mathbf{I}\right)^{2}\right]$ because the trace of $\mathbf{S S}^{\top}$ is fixed at $E=\sum_{k=1}^{M}\left|\mathbf{s}_{k}\right|^{2}$, the total energy in the signal constellation. Thus, in terms of TSC minimization, operations on $\mathbf{R}_{k}$ or $\mathbf{Z}_{k}$ are equivalent.

Note that (27) defines a class of replacement algorithms whereby a given user can reduce (or at least not increase) the total squared correlation assuming other users' codewords remain fixed during the replacement. Such an algorithm may be used by each user sequentially until all users have updated their codewords. At that point the cycle may begin anew. Cycles (iterations) would then be repeated changes in the TSC by individual codeword updates. Consideration of this process begs at least two questions. First, what is an example of such an algorithm? Second, do such algorithms eventually minimize TSC?

In answer to the first question, we present two algorithms. The most obvious method we will call the eigen-algorithm-let $\mathrm{x}=$ $\boldsymbol{\phi}_{k}^{*}$ where $\boldsymbol{\phi}_{k}^{*}$ is a minimum eigenvalue eigenvector of $\mathbf{R}_{k}$. From (15), we see that one step of the eigen-algorithm maximizes the SIR of user $k$ by allowing nonzero signal energy only along

${ }^{3}$ For those unfamiliar with majorization and Schur convexity, an alternate development based on Lagrange methods is provided in [28] and [29]. 
those basis functions with absolute minimum $\lambda_{n}$. From (27), we see that such a choice guarantees $\Delta \geq 0$ (and thereby no increase in the TSC) since both the right and left hand sides of the condition are under-bounded by $\left(\boldsymbol{\phi}_{k}^{*}\right)^{\top} \mathbf{R}_{k} \boldsymbol{\phi}_{k}^{*}$.

We call the less obvious algorithm the MMSE algorithm since we replace $\mathbf{s}_{k}$ by the unit energy MMSE receiver filter $\mathbf{c}_{k}=$ $\left(\mathbf{s}_{k}^{\top}\left[\mathbf{Z}_{k}\right]^{-2} \mathbf{s}_{k}\right)^{-1 / 2}\left[\mathbf{Z}_{k}\right]^{-1} \mathbf{s}_{k}$. We note that in the presence of AWGN, $Z_{k}$ is always invertible. The filter $\mathbf{c}_{k}$ is equivalent to forming $\overline{\mathbf{c}}_{n}=\Lambda^{-1} \overline{\mathbf{s}}$ in the decorrelated space and then renormalizing where $\Lambda$ is the eigenvalue matrix of $Z_{k}$. It is known that codewords might be adapted for single users to obtain better mean square error (MSE) performance [30], [31]. The following theorem, proven in [9], [30], and [32] shows that the MMSE algorithm is indeed an interference avoidance algorithm.

Theorem 1: Replacing $\mathbf{s}_{k}$ with $\mathbf{c}_{k}$ yields $\Delta \geq 0$, with equality iff $\mathbf{c}_{k}=\mathbf{s}_{k}$.

Historically, the MMSE algorithm for codeword ensemble optimization is the first proposed interference avoidance algorithm [9], [32], [33].

The question of convergence is addressed next where we find that the eigen-algorithm and the MMSE algorithm share a number of common properties.

- Both algorithms decrease the TSC monotonically. Since TSC is bounded below by the Welch bound, they must converge in TSC. For the MMSE algorithm, Theorem 1 implies that if TSC converges, then the signatures must converge [32]. For the eigen-algorithm, formal "convergence in class" of codeword ensembles is shown in [29]. At fixed points of both algorithms, each $s_{k}$ is an eigenvector of $\mathbf{Z}_{k}$.

- For neither algorithm is the resulting codeword set unique. For example, any rotation of the codeword set will have the same cross-correlation properties.

- When $M \leq N$, the signatures converge to an orthonormal set. For the MMSE algorithm, this may take several cycles. For the eigen-algorithm, this occurs after one cycle since each user chooses an eigenvector orthogonal to the previously chosen signatures.

- When $M \geq N$, the algorithms may converge to a WBE signature set $\mathbf{S}$ satisfying $\mathbf{S} \mathbf{S}^{\top}=(M / N) \mathbf{I}_{N}$. Alternatively, both algorithms could theoretically converge to a local minimum for TSC. In [9], [32], and [33] mild conditions are derived under which the MMSE algorithm converges. In [34], MMSE interference avoidance is shown to always converge stochastically to the optimum signature set. Empirically, the eigen-algorithm has always converged to minimum TSC in experiments starting from random codeword vectors. In addition, a modification to the procedure called "class warfare" guarantees convergence to a global optimum [28], [29]. Further detail for eigen-algorithm fixed points is provided in Section IV.

The intuition behind all interference avoidance algorithms which obey (27) is embodied by the simple requirement $\mathbf{x}^{\top} \mathbf{R}_{k} \mathbf{x} \leq \mathbf{s}_{k}^{\top} \mathbf{R}_{k} \mathbf{S}_{k}$ - the replacement vector $\mathbf{x}$ attempts to reduce the interference from the ensemble of other user vectors and noise. From the standpoint of implementation, in the MMSE algorithm, user $k$ must identify $\left(\mathbf{R}_{k}+\sigma^{2} \mathbf{I}\right)^{-1} \mathbf{s}_{k}$.
In the eigen-algorithm, user $k$ seeks a minimum eigenvalue eigenvector $\phi_{k}^{*}$ of $\mathbf{R}_{k}$.

These points, taken together suggest that the class of algorithms governed by (27) could be implemented by blind techniques at the receiver along with a feedback channel to the transmitter. Specifically, in the MMSE algorithm, the receiver for user $k$ could be a blind adaptive MMSE filter [35] based on the observable $\mathbf{Z}_{k}=\mathbf{R}_{k}+\sigma^{2} \mathbf{I}$. Likewise, for the eigen-algorithm, $\Delta$ of (27) is maximized when $\mathbf{x}^{\top} \mathbf{R}_{k} \mathbf{x}$, the sum interference experienced by user $k$ with new codeword $\mathbf{x}$, is minimized - equivalent to minimizing $\mathbf{x}^{\top} \mathbf{Z}_{k} \mathbf{x}$. The vector $\mathbf{x}$ can also be found using blind techniques. Thus, interference avoidance algorithms are based on a measurable quantity - the interference/noise signal correlation $\mathbf{Z}_{k}$.

In the MMSE algorithm, a codeword replacement by user $k$ requires first that the receiver filter for user $k$ converges. Further, the MMSE filter coefficients $\mathbf{c}_{k}$ must be communicated to the transmitter via a feedback channel. Consequently, at each iterative step, the speed of the algorithm is limited since: 1) the convergence to the MMSE filter may require several hundred bits and 2) several hundred bits may be needed for the feedback transmission of the new signature. These same conclusions will also hold for the eigen-algorithm. Therefore, these signature adaptation algorithms operate on a slower time scale than the algorithms for multiuser interference suppression. Thus, if the channel is not stable for a sufficient number of bit intervals, it is not clear how much advantage interference avoidance will confer. However, for channels which are stable over a sufficient number of bit intervals, signature adaptation may offer potentially large capacity increases.

\section{FIXED Point Properties FOR GREedy INTERFERENCE AVOIDANCE ALGORITHMS}

Unfortunately, for $M>N$ there is no guarantee that interference avoidance always leads to an optimal fixed point. For example, $\Delta$ from (27) can be zero over a full cycle of an interference avoidance algorithm even though the minimum eigenvalues $\lambda_{k}^{*}$ might not all be equal as required for $\mathbf{S S}^{\top}=(M / N) \mathbf{I}$. An illustration of such a suboptimal signal set is provided in Fig. 1 and the corresponding optimal set in Fig. 2.

We now examine the nature of such suboptimal fixed points in more detail. ${ }^{4}$ The convergence properties of the MMSE algorithm are described in [9] and [32]-[34]. In this paper, our focus will be on the eigen-algorithm, or more generally, on any greedy algorithm which always chooses codeword replacements which increase its SIR when at all possible. Or equivalently, for all interference avoidance algorithms whose only fixed points are those such that the equilibrium codewords for agile users are minimum eigenvalue eigenvectors of their respective $\mathbf{R}_{k}$. This condition leads easily to simple structural conditions on globally optimal algorithm fixed points.

\section{A. Fixed Points for Equal-Power Agile Users}

We start with a Lemma.

\footnotetext{
${ }^{4}$ For simplicity, we assume no uniform background noise, noting that the minimization of TSC is the same whether we consider $\mathbf{R}_{k}$ or $\mathbf{Z}_{k}$.
} 


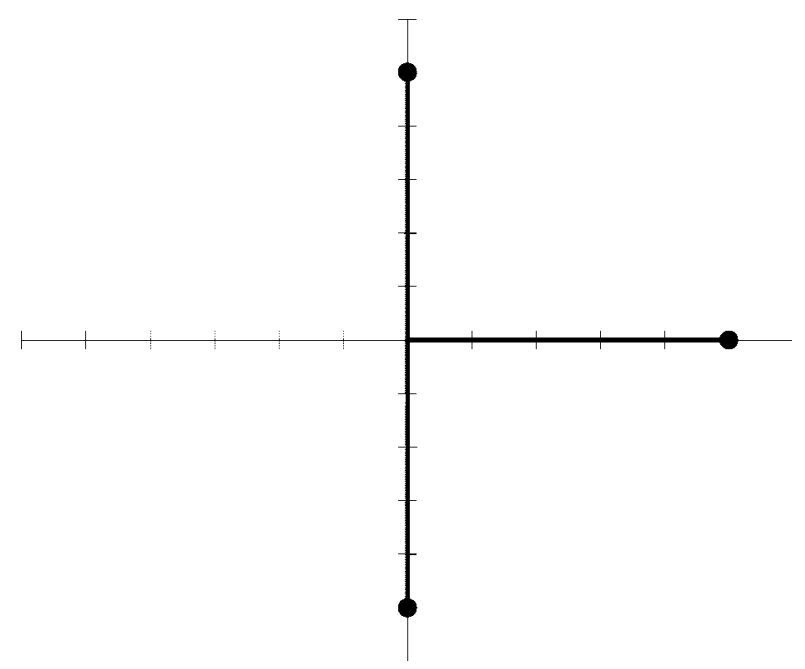

Fig. 1. Simple suboptimal signal set in two dimensions with three users.

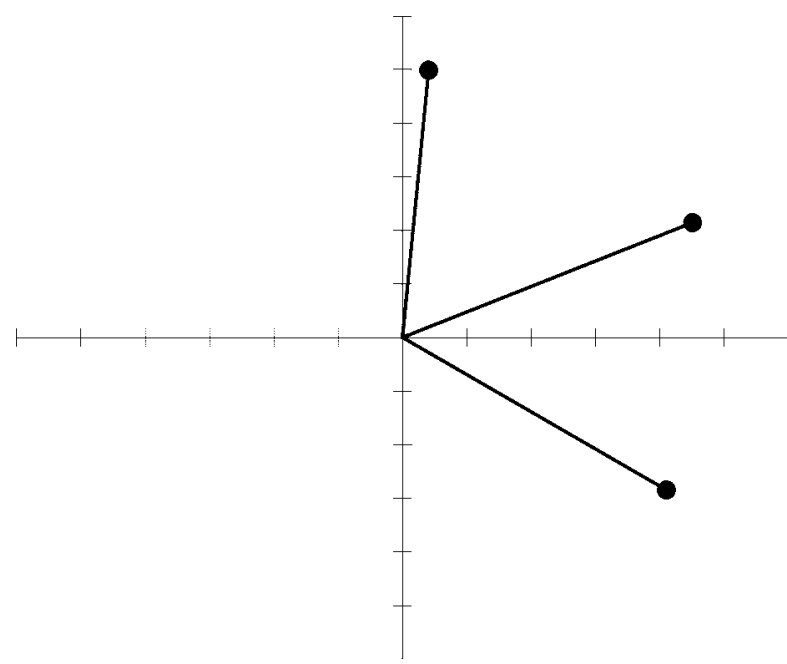

Fig. 2. Simple optimal signal set in two dimensions with three users.

Lemma 1: If $\left\{\mathbf{s}_{k}\right\}$ is a fixed point of a greedy interference avoidance algorithm with minimum eigenvalue ensemble $\left\{\lambda_{k}^{*}\right\}$, the eigenvalue set of each $\mathbf{R}_{k}$ contains $\lambda_{k}^{*}$ and $\left\{\lambda_{j}^{*}+1\right\}$ where $\lambda_{j}^{*} \neq \lambda_{k}^{*}$. Furthermore, all corresponding $\mathbf{s}_{j}$ are also eigenvectors of $\mathbf{R}_{k}$.

Proof: Lemma 1: Since $\lambda_{j}^{*}$ is an eigenvalue of $\mathbf{R}_{j}, \mathbf{R}_{j} \mathbf{s}_{j}=$ $\lambda_{j}^{*} \mathbf{s}_{j}$. Observing that $\mathbf{R}_{j}=\mathbf{R}_{k}+\mathbf{s}_{k} \mathbf{s}_{k}^{\top}-\mathbf{s}_{j} \mathbf{s}_{j}^{\top}$, we have $\mathbf{R}_{k} \mathbf{s}_{j}+$ $\mathbf{s}_{k} \mathbf{s}_{k}^{\top} \mathbf{s}_{j}-\mathbf{s}_{j} \mathbf{s}_{j}^{\top} \mathbf{s}_{j}=\lambda_{j}^{*} \mathbf{s}_{j}$. Both $\mathbf{s}_{k}$ and $\mathbf{s}_{j}$ are eigenvectors of $\mathbf{S S}^{\top}$, but since we assume $\lambda_{j}^{*} \neq \lambda_{k}^{*}$, they must be orthogonal. This implies $\mathbf{R}_{k} \mathbf{s}_{j}=\left(\lambda_{j}^{*}+1\right) \mathbf{s}_{j}$, thus, proving the theorem.

We then use Lemma 1 to show that interference avoidance must produce codeword ensembles which span the signal space as well as provide bounds on the differences in performance between codeword SIRs.

Theorem 2: Let $\left\{\mathbf{s}_{k}\right\}$ be a fixed point with $\mathbf{S S}^{\top}$ having eigenvalues $\left\{\left(\lambda_{k}^{*}+1\right)\right\}$. The distinct eigenvalues, $\lambda_{1}, \ldots \lambda_{p}$ satisfy $\left|\lambda_{i}-\lambda_{j}\right| \leq 1$ for $i, j=1, \ldots, p$. Furthermore, the signal vectors associated with each distinct eigenvalue $\lambda_{i}$ form mutually orthonormal subspaces which collectively span $\Re^{N}$.

Proof: Theorem 2: Each $\mathbf{S}_{k}$ is an eigenvector of $\mathbf{S S}^{\top}$. Assume the eigenvalue of $\mathbf{S S}^{\top}$ associated with $\mathbf{s}_{k}$ is $\lambda_{i}$. In this case, the minimum eigenvalue of $\mathbf{R}_{k}$ must be $\lambda_{k}^{*}=\lambda_{i}-1$. Suppose, without loss of generality, that $\lambda_{i}>\lambda_{j}+1$ for some $j$. This would imply $\lambda_{j}<\lambda_{i}-1=\lambda_{k}^{*}$, which is a contradiction since by Lemma $1, \lambda_{k}^{*}$ is the minimum eigenvalue of $\mathbf{R}_{k}$. For the second part, we note that since $\mathbf{S S}^{\top}$ is symmetric, distinct eigenvalues correspond to orthogonal eigenvectors. Since each of the $\mathbf{s}_{k}$ is also an eigenvector of $\mathbf{S} \mathbf{S}^{\top}$, then the vectors $\mathbf{s}_{k}$ associated with given eigenvalues form mutually orthogonal subspaces of $\Re^{N}$. If these subspaces did not collectively span $\Re^{N}$, then $\mathbf{S S}^{\top}$ would have an eigenvector $\boldsymbol{\phi}$ with zero eigenvalue. This would imply that any signature $\mathbf{s}_{k}$ with $\lambda_{k}^{*}>0$ could be replaced by $\phi$ to reduce TSC, which would be a contradiction. $\bullet$

As an aside which brings closure with the results of [10] and [21], we provide the following simple corollary to Theorem 2.

Corollary 1: Let $M \geq N$. If the set $\left\{\mathbf{s}_{k}\right\}$ comprises a fixed point with $\mathbf{S S}^{\top}$ having eigenvalues $\left(\lambda_{k}^{*}+1\right)=\lambda^{*}$, $k=1,2, \ldots, N$, then $\lambda^{*}=M / N$ and $\mathbf{S S}^{\top}=M / N \mathbf{I}_{N}$.

That is, since any positive semidefinite matrix such as $\mathbf{S S}^{\top}$ is always diagonalizable - as opposed to having a Jordan form [17] - we must have $\mathbf{S} \mathbf{S}^{\top}=\mathbf{Q}\left(\lambda^{*} \mathbf{I}\right) \mathbf{Q}^{\top}=\lambda^{*} \mathbf{Q} \mathbf{Q}^{-1}=\lambda^{*} \mathbf{I}$. Since as noted previously, Trace $\left[\mathbf{S S}^{\top}\right]=M$, we must have $\lambda^{*}=M / N$.

In summary, there are the optimal fixed points with $\lambda_{k}^{*}=M / N-1$ for $k=1, \ldots, M$, and the suboptimal fixed points with vectors $\left\{\mathbf{s}_{\boldsymbol{k}}\right\}$ in mutually orthogonal subspaces corresponding to each different $\lambda_{k}^{*}$ - which cannot differ from each other by more than one. If all the $\lambda_{k}^{*}$ are identical, then the ensemble correlation $\mathbf{S S}^{\top}$ is an identity matrix. That is, the ensemble is essentially whitened.

Our worry, of course, is that the algorithm will converge to a suboptimal point. Fortunately, in all our experience with numerical studies suboptimal minima have never been obtained when the starting ensemble is chosen randomly. Only when the initial set is not full rank or when the component vectors can be partitioned into mutually orthogonal subspaces does the algorithm not always converge to an optimal codeword ensemble.

\section{B. Fixed Points for Mixtures of Fixed and Agile Users}

It is likely that users with both fixed and agile waveform radios may need to occupy the same signal space. We now consider this scenario and find the intuitively pleasing result that using the eigen-algorithm, the agile users perform a sort of $a g$ gregate waterfilling over the portion of the signal space with least fixed user energy to achieve an optimum SIR. Put another way, the fixed users appear as colored noise to the agile users which causes an appropriate Shannonesque distribution of agile user signal energy over the signal space. It is readily understood that this feature is of benefit, on average, to the fixed users as well since it implies that the agile users avoid the fixed users where possible. We also note that the implied minimization of ensemble TSC (including the fixed user signatures) is equivalent to maximization of information theoretic sum capacity [28], [29], [36].

Formally, we let the set $\left\{\mathbf{a}_{k} \mid 1 \leq k \leq L\right\}$ be the set of signal vectors associated with waveform-agile users. Let $\left\{\mathbf{f}_{i} \mid 1 \leq i \leq\right.$ $M-L\}$ be the signal vectors associated with fixed-waveform 
users. Greedy interference avoidance will only be applied to the $\left\{\mathbf{a}_{k}\right\}$. We define

$$
\mathbf{S S}^{\top}=\sum_{k=1}^{L} \mathbf{a}_{k} \mathbf{a}_{k}^{\top}+\sum_{i=1}^{M-L} \mathbf{f}_{i} \mathbf{f}_{i}^{\top}=\mathbf{A} \mathbf{A}^{\top}+\mathbf{F F}^{\top} .
$$

We also define the mutually orthogonal eigenvectors of $\mathbf{F F}^{\top}$ as $\phi_{i}$ with associated eigenvalues $\sigma_{i} \geq 0$.

First consider the case where there are fewer agile users $L$ than dimensions $N$. The agile users cannot span the entire signal space. Thus, we must ask in what portion of the signal space should the agile users reside to achieve minimum interference. The answer, though intuitively obvious, is stated as a theorem and proven.

Theorem 3: If there are $L<N$ agile users, then the interference experienced by at least one agile user can be reduced (while not increasing the interference seen by other agile users) unless the set $\left\{\mathbf{a}_{k}\right\}$ is contained in the space spanned by the $L$ eigenvectors of $\mathbf{F F}^{\top}$ with the smallest eigenvalues.

Note that Theorem 3 can also be restated in terms of TSC since reduction of interference for one user while not increasing that seen by other users clearly reduces TSC.

Proof: Theorem 3: At equilibrium, we have

$$
\left(\mathbf{A A}^{\top}+\mathbf{F F} \mathbf{F}^{\top}\right) \mathbf{a}_{k}=\left(\lambda_{k}^{*}+\left|\mathbf{a}_{k}\right|^{2}\right) \mathbf{a}_{k}
$$

for an interference avoidance algorithm. We note that the dimension of the space in which the $\left\{\mathbf{a}_{k}\right\}$ reside is $Q \leq L<N$, and that we can linearly combine the $\mathbf{a}_{k}$ to derive $Q$ mutually orthonormal eigenvectors of $\mathbf{S S}^{\top}$. However, $\mathbf{S S}^{\top}$ has a full set of eigenvectors so there exists an additional set of $N-Q$ eigenvectors each of which is orthogonal to the $\left\{\mathbf{a}_{k}\right\}$. If $\mathbf{x}_{i}$, $i=1, \ldots, N-Q$ is one such eigenvector, then it must also be an eigenvector of $\mathbf{F F}^{\top}$ since

$$
\mathbf{A A}^{\top} \mathbf{x}_{i}+\mathbf{F F}^{\top} \mathbf{x}_{i}=\mathbf{F} \mathbf{F}^{\top} \mathbf{x}_{i}=\eta_{i} \mathbf{x}_{i}
$$

With no loss of generality, let us assume that the first $N-Q$ eigenvectors of $\mathbf{F F}^{\top}$, are exactly these $\mathbf{x}_{i}$ and that the corresponding eigenvalues $\sigma_{i}$ are exactly the $\eta_{i}{ }^{5}$ Since the eigenvectors $\phi_{i}$ collectively span $\Re^{N}$ and the eigenvectors $\phi_{i} i=$ $1, \ldots, N-Q$ comprise the orthogonal complement of the $\left\{\mathbf{a}_{k}\right\}$, we can expand the $\left\{\mathbf{a}_{k}\right\}$ in $\boldsymbol{\phi}_{j}, j=N-Q+1, \ldots, N$ as

$$
\mathbf{a}_{k}=\sum_{i=1}^{Q} \alpha_{i}(k) \phi_{N-Q+i}
$$

with $\alpha_{i}(k)=\mathbf{a}_{k}^{\top} \boldsymbol{\phi}_{N-Q+i}$.

The interference experienced by user $k$ is $\lambda_{k}^{*}$ which we can write as

$$
\left(\lambda_{k}^{*}+\left|\mathbf{a}_{k}\right|^{2}\right)\left|\mathbf{a}_{k}\right|^{2}=\mathbf{a}_{k}^{\top}\left(\mathbf{A} \mathbf{A}^{\top}+\mathbf{F F}^{\top}\right) \mathbf{a}_{k} .
$$

Using (31), we obtain after some simplification

$$
\begin{aligned}
\left(\lambda_{k}^{*}+\left|\mathbf{a}_{k}\right|^{2}\right)\left|\mathbf{a}_{k}\right|^{2}= & \sum_{\ell=1}^{L}\left[\sum_{i=1}^{Q} \alpha_{i}(k) \alpha_{i}(\ell)\right]^{2} \\
& +\sum_{i=1}^{Q} \alpha_{i}^{2}(k) \sigma_{N-Q+i} .
\end{aligned}
$$

${ }^{5}$ We then note that $\phi_{j}$ might not be an eigenvector of $\mathbf{S S}^{\top}$ for $j=N-Q+$ $1, \ldots, N$.
Now suppose that for some $\ell=1, \ldots, N-Q$ and $m=$ $N-Q+1, \ldots, N$, we have $\sigma_{m}>\sigma_{\ell}$. For the $\alpha_{i}(k)$ fixed, if we swap $\boldsymbol{\phi}_{\ell}$ with $\boldsymbol{\phi}_{m}$, the only term in (33) which can change is the second sum on the right-hand side. Since this substitution cannot increase any of the $\sigma_{N-Q+i}$ we will not increase any $\lambda_{k}^{*}$. In fact, we will reduce $\lambda_{k}^{*}$ for at least one value of $k$ since there must exist some $k$ for which $\alpha_{m}(k) \neq 0$ by the condition that the space spanned by the $\left\{\boldsymbol{\phi}_{i}\right\}, i=N-Q+1, \ldots, N$ and the space spanned by the $\left\{\mathbf{a}_{k}\right\}$ are coincident.

Therefore, unless $\sigma_{m} \leq \sigma_{\ell}$ for $\ell=1, \ldots, N-Q$ and $m=N-Q+1, \ldots, N$, the interference experienced by at least one of the agile users can be reduced by the substitution of basis vectors described above without increasing the interference seen by the other agile users. Therefore, to achieve minimum mutual interference, the $\left\{\mathbf{a}_{\boldsymbol{k}}\right\}$ must reside in the space spanned by the $Q \leq L$ eigenvectors of $\mathbf{F F}^{\top}$ with smallest eigenvalues. Since this space is contained in the space spanned by the $L$ eigenvectors of $\mathbf{F F}^{\top}$ with smallest eigenvalues, the theorem is proved.

We can, therefore, assume with no loss of generality that $L \geq$ $N$ - since if not, we simply recast the problem in a space of dimension $N^{\prime}=L$ following Theorem 3 . We now provide a bound for the minimum TSC.

Theorem 4: When $\mathbf{S S}^{\top}=\mathbf{A} \mathbf{A}^{\top}+\mathbf{F F}^{\top}$ with $\mathbf{F}$ fixed

$$
\operatorname{Trace}\left[\left(\mathbf{S S}^{\top}\right)^{2}\right] \geq\left(N-h^{*}\right)\left(\frac{M-\sum_{i=1}^{h^{*}} \sigma_{i}}{N-h^{*}}\right)^{2}+\sum_{i=1}^{h^{*}} \sigma_{i}^{2}
$$

where the $\left\{\sigma_{i}\right\}$ are the eigenvalues of $\mathbf{F F}^{\top}$ ordered from largest to smallest, and $h^{*}=\arg \min _{h}\left(M-\sum_{i=1}^{h} \sigma_{i}\right) /(N-h)$. This bound is met with equality when the eigenvalues of $\mathbf{S S}^{\top}$ are $\left\{\sigma_{1}, \ldots, \sigma_{h^{*}}, c^{*}, \ldots, c^{*}\right\}$ where $c^{*}=\left(M-\sum_{i=1}^{h^{*}} \sigma_{i}\right) /(N-$ $h^{*}$ ) has multiplicity $N-h^{*}$.

Proof: Theorem 4: First we write $\mathbf{S S}^{\top}$ in terms of the eigenvectors of $\mathbf{F F}^{\top}$ with $\mathbf{a}_{k}=\sum_{i=1}^{N} \alpha_{i}(k) \boldsymbol{\phi}_{i}$ and $\mathbf{f}_{m}=$ $\sum_{i=1}^{N} \gamma_{i}(m) \phi_{i}$. Using (28), we have

$$
\mathbf{S S}^{\top}=\sum_{i, j=1}^{N}\left(\sum_{k=1}^{L} \alpha_{i}(k) \alpha_{j}(k)+\sum_{\ell=1}^{M-L} \gamma_{i}(\ell) \gamma_{j}(\ell)\right) \boldsymbol{\phi}_{i} \boldsymbol{\phi}_{j}^{\top} .
$$

Remembering that $\boldsymbol{\phi}_{i}^{\top} \boldsymbol{\phi}_{j}=\operatorname{Trace}\left[\boldsymbol{\phi}_{i} \boldsymbol{\phi}_{j}^{\top}\right]=\delta_{i j}$, we form $\left(\mathbf{S S}^{\top}\right)^{2}$ and then take the trace to obtain

$\operatorname{Trace}\left[\left(\mathbf{S S}^{\top}\right)^{2}\right]=\sum_{i, j=1}^{N}\left(\sum_{k=1}^{L} \alpha_{i}(k) \alpha_{j}(k)+\sum_{\ell=1}^{M-L} \gamma_{i}(\ell) \gamma_{j}(\ell)\right)^{2}$.

We define the agile energy contained in $\phi_{j}$ as $\mu_{j}=$ $\sum_{k=1}^{L} \alpha_{j}^{2}(k)$ and note that $\sum_{j=1}^{N} \mu_{j}=L$ the total agile signal energy. The corresponding energy in the fixed signals is $\sigma_{j}=\sum_{\ell=1}^{M-L} \gamma_{j}^{2}(\ell)$ by the definition of the $\phi_{i}$. We can then rewrite (36) as

$$
\begin{aligned}
\operatorname{Trace}\left[\left(\mathbf{S S}^{\top}\right)^{2}\right]= & \sum_{\substack{i \neq j \\
i, j=1}}^{N}\left(\sum_{k=1}^{L} \alpha_{i}(k) \alpha_{j}(k) \sum_{\ell=1}^{M-L} \gamma_{i}(\ell) \gamma_{j}(\ell)\right)^{2} \\
& +\sum_{j=1}^{N}\left(\mu_{j}+\sigma_{j}\right)^{2}
\end{aligned}
$$


The terms in the left-hand sum are nonnegative, thus, Trace $\left[\left(\mathbf{S S}^{\top}\right)^{2}\right] \geq \sum_{j=1}^{N}\left(\mu_{j}+\sigma_{j}\right)^{2}$, which is convex in the $\mu_{j}$. Since we require $\mu_{j} \geq 0$ and $\sum_{j=1}^{N} \mu_{j}=L$, application of standard constrained optimization techniques [37]-[39] provides a classical waterfilling result. Defining the "water level" as $c^{*}=\left(M-\sum_{i=1}^{h^{*}} \sigma_{i}\right) /\left(N-h^{*}\right)$ with $h^{*}$ as defined in the theorem statement, we have $\mu_{j}=0$ when $\sigma_{j}>c^{*}$ and $\mu_{j}=c^{*}-\sigma_{j}$ otherwise. Thus

$$
\operatorname{Trace}\left[\left(\mathbf{S S}^{\top}\right)^{2}\right] \geq\left(N-h^{*}\right)\left(c^{*}\right)^{2}+\sum_{i=1}^{h^{*}} \sigma_{i}^{2}
$$

thereby, proving the first part of the theorem. Now, Trace $\left[\left(\mathbf{S S}^{\top}\right)^{2}\right]=\sum_{i=1} \lambda_{i}^{2}$ where the $\lambda_{i}$ are eigenvalues of $\mathbf{S S}^{\top}$. Using the eigenvalues for $\mathbf{S S}^{\top}$ defined in the theorem statement completes the proof.

Experimentally, for randomly chosen initial codeword vectors $\left\{\mathbf{a}_{k}\right\}$ and $\left\{\mathbf{f}_{k}\right\}$, invariably the lower bound of Theorem 4 was attained. Analytically, the existence of such signal sets is guaranteed by application of a variant of the eigen-algorithm [28], [29]. As for agile-only users, a modification to the algorithm guarantees convergence to the optimum. Thus, greedy interference avoidance algorithms seek a minimum mutual interference set of agile vectors by "water filling" the energy levels provided by the fixed users, and avoiding completely energetic interference above a certain threshold.

Also note that as a byproduct, we have also shown that the algorithm seeks a minimum mutual interference set of vectors in a background of colored noise. That is, the effect of the fixed users came into play only through the eigenvalues and eigenvectors of $\mathbf{F F}^{\top}$ which could be considered as the autocorrelation matrix of some arbitrary colored noise process.

Finally, we note that as in the agile-only case, there are optimal fixed points where the agile users obtain uniformly maximum SIR, and suboptimal fixed points where groups of different agile users obtain differing SIRs. In the suboptimal case, it is easily shown that once again, the agile users are partitioned into mutually orthogonal subspaces according to the SIR obtained. Fortunately, also as with the agile-only case, random choice of initial vectors $\left\{\mathbf{a}_{k}\right\}$ precluded convergence to a suboptimum minimum in all our numerical experiments.

\section{Fixed Points for Interference Avoidance With Unequal Power}

In this section, we consider the case where each user has arbitrary but fixed received power $p_{k}$. We will find that interference avoidance achieves eigenvalues for $\mathbf{S S}^{\top}$ identical to those shown in [26] to maximize the sum capacity. We explicitly add white Gaussian background noise so that capacity is well-defined although this is not a necessary feature for interference avoidance to be effective. We remind the reader that we assume at least as many users $M$ as signal dimensions $N$ since in the event $M<N$, the users will confine themselves to $M$ dimensions. Note also once again that we have incorporated the signal power $p_{k}$ into the code vector $\mathbf{s}_{k}$; i.e., $\left|\mathbf{s}_{k}\right|^{2}=p_{k}$.

In an AWGN background, at equilibrium, we require

$$
\left(\mathbf{S S}^{\top}-\mathbf{S}_{k} \mathbf{S}_{k}^{\top}+\sigma \mathbf{I}\right) \mathbf{s}_{k}=\left(\lambda_{k}^{*}+\sigma\right) \mathbf{s}_{k}
$$

With $p_{k}=\left|\mathbf{s}_{k}\right|^{2}$, we have

$$
\left(\mathbf{S S}^{\top}+\sigma \mathbf{I}\right) \mathbf{s}_{k}=\left(\left[\lambda_{k}^{*}+\sigma\right]+p_{k}\right) \mathbf{s}_{k}=p_{k}\left(\beta_{k}^{*}+1\right) \mathbf{s}_{k} .
$$

The quantity $\beta_{k}^{*}=\lambda_{k}^{*}+\sigma / p_{k}$ is the inverse of the signal-to-interference-plus noise ratio (SINR) achieved by the $k^{\text {th }}$ user. We choose $\beta_{k}^{*}$ as small as possible which in turn implies we choose the minimum eigenvalue eigenvector of $\mathbf{S S}^{\top}$ as previously discussed. We also have Trace $\left[\mathbf{S S}^{\top}\right]=E$ where $E=\sum_{j=1}^{M} p_{k}$. Likewise, Trace $\left[\left(\mathbf{S S}^{\top}\right)^{2}\right]$ is not increased by an interference avoidance algorithm which replaces $\mathbf{s}_{k}$ with an $\mathbf{x}$ of equal power [see (26)].

Since the interference avoidance algorithm cannot increase Trace $\left[\left(\mathbf{S S}^{\top}\right)^{2}\right]$, we now seek a lower bound for Trace $\left[\left(\mathbf{S S}^{\top}\right)^{2}\right]$. We have Trace $\left[\left(\mathbf{S S}^{\top}\right)^{2}\right]=\sum_{n=1}^{N} \kappa_{n}^{2}$ where the $\left\{\kappa_{n}\right\}$ are the eigenvalues of $\mathbf{S S}^{\top}$. Consider then that for any signal with power $p_{k}$, the corresponding eigenvalue of $\mathbf{S} \mathbf{S}^{\top}$ is at least $p_{k}$. This forms the basis for the constraint on the eigenvalues of $\mathbf{S S}^{\top}$ mentioned in Section III-A and is identical to the constraint on the $\lambda_{i}$ derived for sum capacity in [26]. That is, $\mathbf{S S}^{\top} \mathbf{s}_{k}=\mathbf{R}_{k} \mathbf{s}_{k}+\mathbf{s}_{k} \mathbf{s}_{k}^{\top} \mathbf{s}_{k}=\left(\lambda_{k}^{*}+p_{k}\right) \mathbf{s}_{k}$ and we have in general

$$
\operatorname{Trace}\left[\left(\mathbf{S S}^{\top}\right)^{2}\right]=\sum_{i=1}^{N}\left(c_{i}+p_{i}\right)^{2}
$$

with $c_{i} \geq 0$ and assuming ordered energies, $p_{i} \geq p_{i+1}$. Further assuming ordered eigenvalues $\kappa_{i} \geq \kappa_{i+1}$ we have $\kappa_{i} \geq p_{i}$, $i=1,2, \ldots, N$.

Since the eigenvalues must sum to $E$, we have $\sum_{i=1}^{N}\left(c_{i}+\right.$ $\left.p_{i}\right)=E$. The minimization of Trace $\left[\left(\mathbf{S S}^{\top}\right)^{2}\right]$ requires $c_{i}=0$ if $p_{i}>c^{*}$ and $c_{i}=c^{*}-p_{i}$ when $p_{i} \leq c^{*}$. Once again, the "water level" $c^{*}$ which satisfies the power constraint equation is

$$
c^{*}=\frac{E-\sum_{i=1}^{h^{*}} p_{i}}{N-h^{*}}
$$

where

$$
h^{*}=\arg \min _{h}=\frac{E-\sum_{i=1}^{h} p_{i}}{N-h} .
$$

Thus, $c^{*}$ is an eigenvalue of $\mathbf{S} \mathbf{S}^{\top}$ with multiplicity $N-h^{*}$ and the complete set of eigenvalues of $\mathbf{S} \mathbf{S}^{\top}$ is

$$
\left\{p_{1}, \ldots, p_{h^{*}}, c^{*}, \ldots, c^{*}\right\} .
$$

From (40), we see that

$$
\beta_{k}^{*}= \begin{cases}\frac{\sigma}{p_{k}}, & k \leq h^{*} \\ \frac{\left(\sigma+c^{*}-p_{k}\right)}{p_{k}}, & h^{*}<k \leq M .\end{cases}
$$

If no $p_{k}>E / N$, we then have the usual uniform solution of $c^{*}=E / N\left(h^{*}=0\right)$ and consequently

$$
\beta_{k}^{*}=\frac{\sigma}{p_{k}}+\frac{E}{p_{k} N}-1 .
$$

Overall, these results have a facetious but memorable interpretation Might makes right. That is, those users with greater received power $p_{k}$ obtain better performance. In fact, the excessively energetic $h^{*}$ users command private channels. Such users are called oversized in [26], a term coined by S. Verdu. 


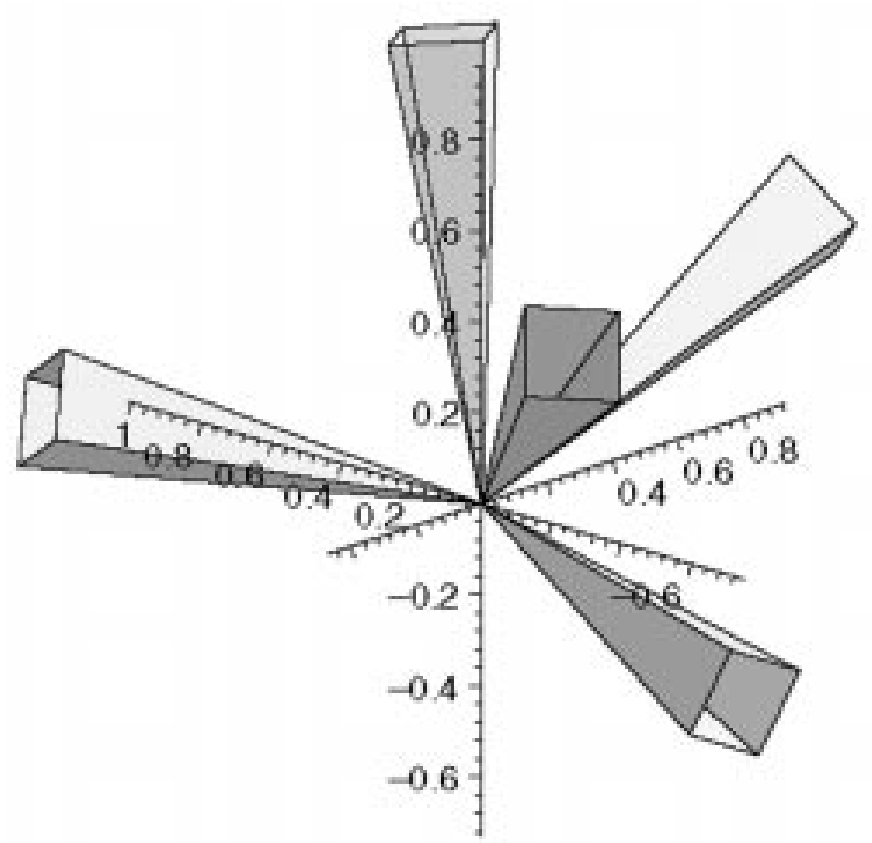

Fig. 3. Vector plot of five signal vectors in three-space after five cycles of interference avoidance. Signal vectors are represented as hollow inverted pyramids for greater clarity of the 3-dimensional (3-D) representation.

It is worth noting again that minimizing Trace $\left[\left(\mathbf{S S}^{\top}\right)^{2}\right]$ also minimizes Trace $\left[\left(\mathbf{S S}^{\top}+\sigma \mathbf{I}_{N}\right)^{2}\right]$. Since minimizing Trace $\left[\left(\mathbf{S S}^{\top}+\sigma \mathbf{I}_{N}\right)^{2}\right]$ under constraints on the eigenvalues is equivalent to maximizing the sum capacity (see Section III-A) under the same constraints, the eigenvalues of $\mathbf{S S}^{\top}$ given in (44) imply a codeword set which can achieve the sum capacity. Furthermore, when no $p_{k}>E / N$, we have $h^{*}=0$ and $\lambda_{k}^{*}=\lambda^{*} \forall k$. This implies an absolute minimum TSC which in turn implies an absolute maximum sum capacity. This result is in agreement with that provided in [26] where an existence proof for such codeword sets can also be found.

Finally we note, once again, that suboptimal minima may be obtained in which users are partitioned into mutually orthogonal subspaces with differing SINR characteristics. Also as before, these subpoptimal fixed points seem to be avoided by starting from initially random codewords and that convergence to optimum can be forced by a modification of the greedy interference avoidance procedure [28], [29].

\section{DISCUSSION}

\section{A. Eigen-Algorithm: Numerical Examples}

Here, we provide example applications of interference avoidance to the scenarios analyzed in this paper. Fig. 3 shows CDMA chip sequences for five agile users with three chips after five interference avoidance cycles. The resultant associated $\mathbf{S S}^{\top}$ is approximately diagonal

$$
\mathbf{S S}^{\top}=\left[\begin{array}{ccc}
1.667 & -0.00035 & -0.00002 \\
-0.00035 & 1.666 & 0.00017 \\
-0.00002 & 0.00017 & 1.666
\end{array}\right]
$$

and each unit energy user achieves a signal to interference ratio of approximately $N / M-N=1.5$.

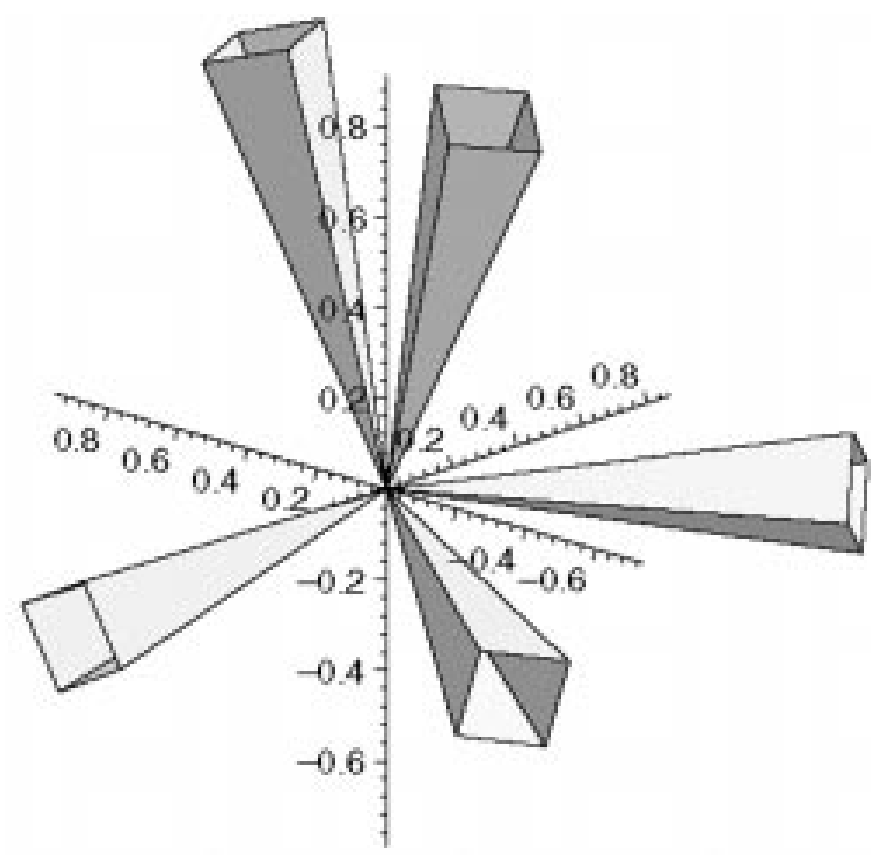

Fig. 4. Vector plot of five signal vectors (three agile and two fixed) after five cycles of interference avoidance. Notice the coplanarity of the three agile signal vectors in avoidance of a strong fixed interferer component along the remaining dimension.

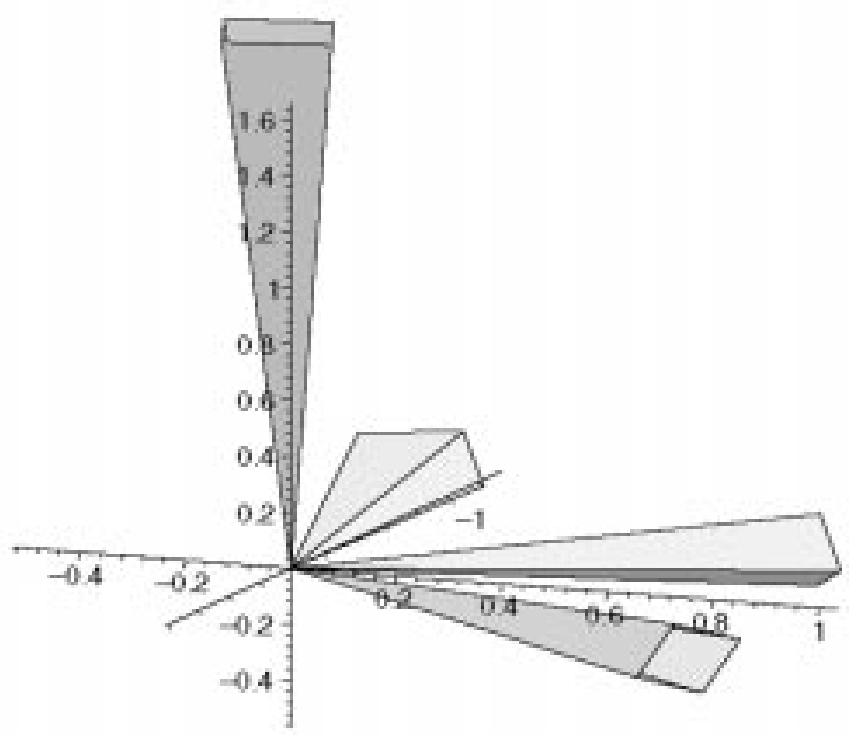

Fig. 5. Vector plot of four signal vectors, three with power 1 and the remaining with power 4 . Notice the coplanarity of the three power 1 vectors in avoidance of the strong interferer with power 4 .

In Fig. 4, we allow only the first three signals to be agile and fix the remaining two. After five cycles the three agile users reside in a space of dimension 2 (coplanar) achieving approximately $\lambda^{*}=1.6$ and a concomitant signal to interference ratio of approximately $\left(\lambda^{*}-1\right)^{-1}=1$. 66. They avoid a strong fixed user interference component (with energy 1.8) in the remaining dimension.

In Fig. 5, we assume four users, one of whom has much larger power than the others $\left(p_{1}=4\right)$. This energetic user commands a private channel and the remaining three users are forced to share 


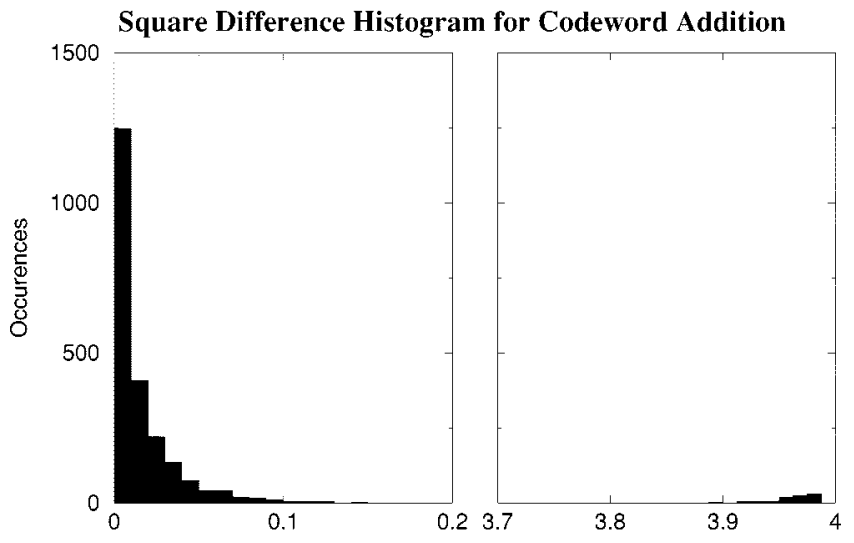

Fig. 6. Histogram of norm squared difference between initial and convergent waveforms after addition of a single new user to an optimal signal set of 24 users in 16 signal dimensions. The central interval $(0.2,3.7)$ over which there were no occurrences was compressed for clarity.

two dimensions of the signal space - again the weaker users are coplanar - and have $\lambda^{*}=3 / 2$ for a shared SIR of two.

\section{B. Addition and Deletion of Users: Convergence Speed and Codeword Stability}

We have not conducted rigorous convergence-speed experiments for greedy interference avoidance algorithms. For the MMSE algorithm, however, the settling time seems rapid [9], [33] with near-convergence to minimum TSC within a three or four iteration cycles. In 100 trials using $M=24$ and $N=$ 16 , the evolution of Trace $\left[\left(\mathbf{S S}^{\top}\right)^{2}\right]$ for the eigen-algorithm also converged within a few iteration cycles.

However, much more interesting from a system standpoint is the issue of what occurs when a given set of users are disturbed by the addition or deletion of a user. How rapidly does the system settle after the perturbation? Perhaps even more important is the question of how much the signatures change since large signature adjustments may imply large signaling load on the codeword feedback channel.

After one hundred trials where a single user was either added to or deleted from an already settled system with 24 users in 16 dimensions, we found that convergence was once again rapid and occurred within three ensemble waveform update cycles. The magnitude squared of the difference between the preaddition/deletion codewords and the final convergent codewords was used as a measure of codeword volatility. A square difference of zero or four implies respectively, identical initial and final codewords, or codewords which differ only in sign. A square difference of two implies a final codeword orthogonal to the initial.

The results are plotted as histograms in Figs. 6 and 7. As can be seen, the post deletion codewords are more volatile than the post addition codewords. However, neither convergent set often differs much from their preaddition/deletion counterparts. Where they do differ significantly (square difference approximately four), the original codeword has simply been inverted. We believe this to be an artifact of the manner in which eigenvectors are chosen for the eigen-algorithm although we have not pursued the issue carefully. Regardless, these limited results
Square Difference Histogram for Codeword Deletion

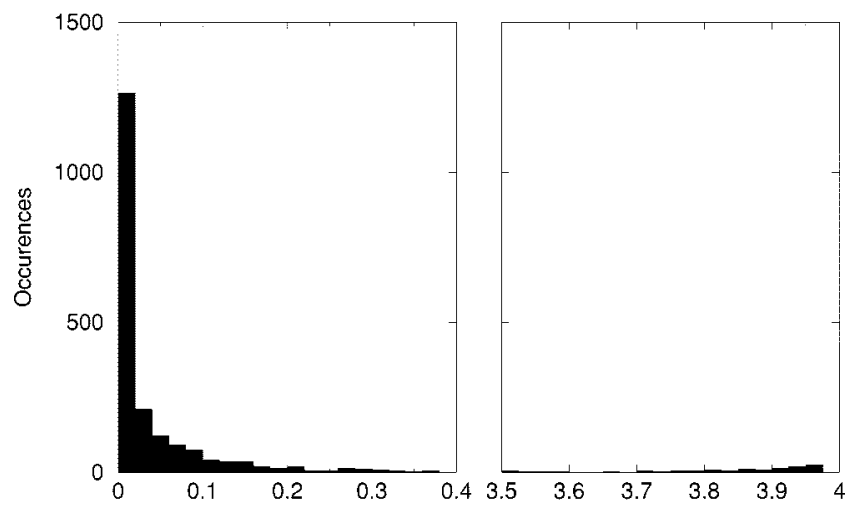

Fig. 7. Histogram of norm squared difference between initial and convergent waveforms after deletion of a single user from an optimal signal set of 24 users in 16 signal dimensions. The central interval $(0.4,3.5)$ over which there were no occurrences was compressed for clarity.

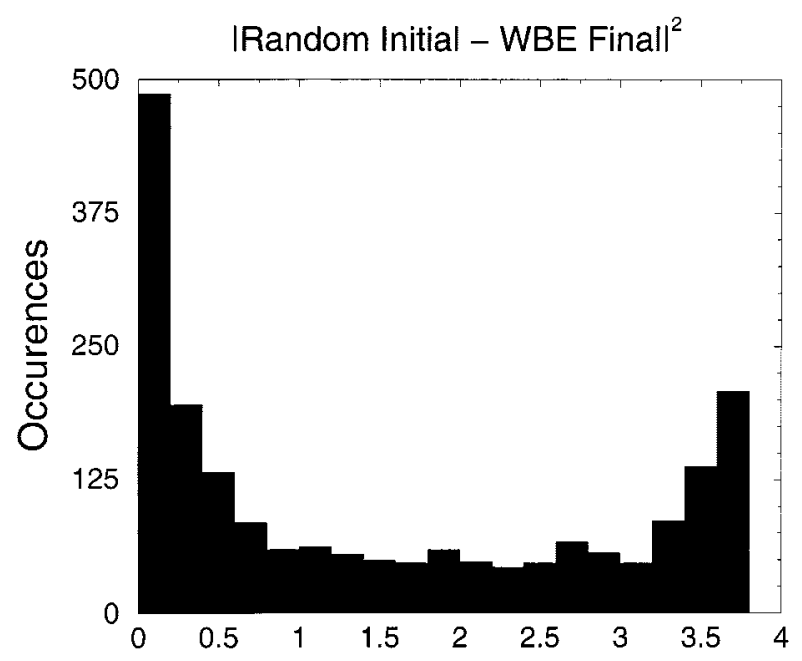

Fig. 8. Histogram of norm squared difference between initial and convergent waveforms for 24 users in 16 signal dimensions.

seem to indicate that codeword adaptation under arrivals and departures could be more orderly and rapid than might initially be imagined.

For comparison, we also provide a histogram (Fig. 8) which shows the difference between initial random codewords and the WBE-converged ensemble. Note that the difference between initial and final codewords is much more pronounced than in Figs. 6 or 7. However, it is also apparent that there are also many codewords which do not differ greatly from their initial randomly chosen value $(\approx 0)$, or only in sign $(\approx 4)$. Thus, even during system startup, the amount of feedback to transmitters might not be excessive.

We close this section with a caveat. Studies of convergence rate based on perfect information are chimeric. In practical systems where information is imperfect, stochastic convergence studies [8] are necessary, and as a general rule, the convergence rate is much slower. Nonetheless, perfect information can often be used to suggest an upper bound on convergence properties. That is, were convergence slow and codewords volatile, there would be little hope of rapid and stable behavior under imperfect information. However, it should also be noted 
that imperfect measurements tend to randomize the codeword replacement process slightly which, following our results for random initial codewords, would tend to favor convergence to the optimum. In fact, it is exactly this property which guarantees MMSE interference avoidance convergence to optimal [34].

\section{A Simple Comparison to S-CDMA}

Suppose that in an interference limited system (assume no background noise) a signal to interference ratio of $\gamma$ is required for each user. The maximum number of users which can be supplied the requisite $\gamma$ is called the user capacity of the system [10]. The work presented, thus, far begs a comparison to CDMA systems since the basic ideas - spreading users over the signal space - are very similar.

Since exact comparisons are difficult even between systems with known operating characteristics (i.e., the raging debate between TDMA and CDMA), we opt for the high road and consider only simple measures for comparison. Furthermore, we will ignore such issues as relative immunity to various channel impairments. We will also not attempt to make estimates of receiver complexity in any real sense since with changing technology, what is complex today may be standard tomorrow. The most obvious comparison is single-base system capacity. Although it is well known that CDMA is inefficient in this regard and we all expect almost any system which attempts to orthogonalize user signatures to be far superior, the single-base comparison will aid us in comparing simple measures of multibase performance.

For a synchronous CDMA (S-CDMA) system with randomly chosen $N$-chip signature sequences, the user capacity with simple matched filters is upper bounded by $N / \gamma$ [40]. For a system employing interference avoidance with matched filters, we have determined that at convergence, the user capacity is $N(\gamma+1) / \gamma$. The ratio of user capacity of interference avoidance to S-CDMA systems is, therefore, at least

$$
\eta=\gamma+1
$$

We note that this improvement is identical to that obtained where MMSE filters as opposed to matched filters are used with random codewords [40].

For $\gamma=1(0 \mathrm{~dB})$ the gain is approximately two. For $\gamma=4$ $(6 \mathrm{~dB})$, the gain is approximately five. Thus, as expected, signatures generated by interference avoidance greatly increase the single-base user capacity of S-CDMA systems. Furthermore, the basic receiver structure (again, conveniently excluding the machinery necessary to calculate and disseminate the signatures) is conceptually simple - a matched filter.

Of course, it would be more interesting to compare the multibase performance of these systems. The current thinking is that although single-base CDMA is inefficient, when multiple contiguous coverage regions are added, CDMA is more efficient and robust than other modulation methods such as TDMA or FDMA. The details of any rigorous comparison lie outside the scope of this paper, so again we opt for simplicity.

Thus, consider an S-CDMA system model where each cell carries $M$ users and the total other-cell interference energy for any given cell is $M \nu / N$ per dimension where $\nu$ is some nonnegative constant. That is, we approximate all the interference energy from all other cells as white over the signal space with aggregate interference energy $M \nu$.

The SIR for each user assuming matched filters and random signatures is

$$
\gamma=\frac{1}{\frac{(M-1)}{N}+\frac{M \nu}{N}}=\frac{N}{(\nu+1) M-1}
$$

for a user capacity of

$$
M_{\mathrm{CDMA}}=\frac{\frac{N}{\gamma}+1}{\nu+1}=\frac{N+\gamma}{\gamma(\nu+1)} .
$$

Unfortunately, a direct comparison to a system employing interference avoidance is as yet impossible since the multiple base interference avoidance problem has not yet been solved. However, we note that for a given cell with other-cell noise energy $M \nu$, the worst interference spectral structure from a capacity standpoint would be white since interference avoidance could exploit the structure of nonwhite interference. Thus, taking on faith (and limited empirical observations under specific scenarios) that fixed points indeed exist for multiple base systems employing interference avoidance, we see that at each base, the "worst case" attainable interference-to-signal ratio (ISR) is $M+\nu M / N-1$ which implies

$$
M_{\mathrm{IA}}=\frac{N(\gamma+1)}{\gamma(\nu+1)}
$$

-an expected improvement of $(1+\gamma) /(1+\gamma / N)$ over S-CDMA.

This approximate comparison, even if flawed by the unknown behavior of interference avoidance in a multiple base setting, suggests that exploration of interference avoidance as a means to increase system capacity will merit further investigation. Of particular interest will be some of the issues raised previously, principal among them, whether interference avoidance can be used effectively with dispersive channels. Recent work [41] where a version of multicarrier modulation is adopted suggests the answer is yes.

\section{SUMMARY AND CONCLUSION}

Starting from a general signal space foundation, we have derived a class of interference avoidance algorithms whereby individual users asynchronously adjust their transmitted waveforms and corresponding matched filter receivers to minimize interference from other sources, including other users. This method presupposes that transmitters and receivers are waveform agile as would be the case assuming universal radios [1]-[5] are inexpensive and ubiquitous.

The interference avoidance procedure, based on SIR as opposed to individual capacity maximization, minimizes total square correlation (TSC). We pursued the relation between sum capacity maximization [10], [25], [26] and minimization of TSC and found equivalence (see Section III-A) in a white noise environment. Specifically, minimization of total square correlation results in signature sets which can also be used to achieve sum channel capacity (through Gaussian signaling).

The minimization/maximization is often achieved through a satisfyingly information theoretic "waterfilling" of the signal space by the ensemble of users. Note that this waterfilling is 
essentially an "emergent" property of the ensemble since individual users seek not to maximize their capacity (via waterfilling), but rather, only seek to maximize their own SINR. Combinations of fixed and agile users (or agile users in colored background noise, see Section IV-B) as well as users with unequal received powers (Section IV-C) were analyzed as examples.

Numerical experiments were conducted which corroborated the basic analytic results. Further experiments, aimed at determining whether interference avoidance might be used in practical systems were also conducted. These included measurements of convergence speed and codeword set volatility under the addition or deletion of a single user. In all cases the results were encouraging. It is also worth noting that experimentally, suboptimal sets were never obtained when starting from randomly chosen codewords and no modifications of the basic interference avoidance procedure (as in [28] and [29]) were necessary.

Simple comparisons to synchronous CDMA show that for a single cell, the number of users serviceable at reasonable SIRs is much greater and scales approximately with the required SIR. This is not surprising since almost any scheme which attempts to orthogonalize signature waveforms will have much greater capacity than a single-base CDMA system. More telling is the comparison of systems for multiple bases where rough calculations suggest substantial improvement may be had using interference avoidance methods, although these results must be interpreted in light of the fact that the behavior of multiple base interference avoidance is as yet unknown. Furthermore, aside from the assumption of waveform agility and the necessity of measuring interference to calculate the optimal signature, the optimum linear transmit and receive methods are very simple - matched filter detection.

We close with a (nonexhaustive) sampling of problem areas, both theoretical and practical, which must be addressed if interference avoidance is to become a useful tool for wireless system design. First, the issue of the proper venue for interference avoidance must be established. By virtue of the necessity for interference measurement over a large number of bit intervals for each waveform update and the asynchronicity of the algorithm over multiple users, it is not obvious whether interference avoidance can be effective in situations where the wireless channel is changing significantly over, say 1000 or 10000 , bit intervals, though there are hints that even in such circumstances, IA affords some improvement [42]. Regardless, a fixed wireless environment might be most appropriate for application of interference avoidance methods.

We note that despite the assumption of perfect channels used in this paper, wireless channels are notoriously dispersive, and when signal energy for each bit is not contained within a single received bit interval, the problem formulation changes. Nonetheless, recent results show that interference avoidance methods seem to be robust and are applicable to dispersive systems, asynchronous systems and multiple antenna systems as well [41]-[50].

As noted, we have found the speed and stability with which codeword ensembles converge surprising. Especially intriguing is the seemingly certain convergence to an optimal set when starting from a randomly chosen starting set and the ease with which suboptimal fixed points are escaped through perturbation. It has been shown that for MMSE interference avoidance this property is a result of stochastic convergence and that MMSE interference avoidance must converge with probability 1 to optimal fixed points in the cases considered [34]. In fact, a modification of greedy interference avoidance called "class warfare" can be used to escape the finite number of local minima and results in provable convergence to minimum TSC [28], [29]. But frustratingly, a general proof for greedy interference avoidance which reflects the uncannily uniform convergence seen in practice without escape methods remains elusive.

The issue of codeword representation and fidelity in a real software/universal radio has been considered only empirically at present [51], [52]. Specifically, instead of uniform-amplitude codeword "chips," interference avoidance presumes real-valued "chips" or more generally, real-valued coefficients for a set of orthonormal signal basis functions used by the transmitter and receiver. To be effective, these values must be communicated to the transmitter, and the amount of information which may be fed back to the transmitter will determine quantization methods for codewords. It is not currently known how quantization affects the performance of interference avoidance, although empirical investigation seems to indicate that in the vecinity of four-bits per chip using Gaussian quantization is sufficient.

Finally, it must be emphasized that only a single receiver (or colocated multiple receivers) were assumed for this study. Limited experiments with multiple receivers and power control showed unstable behavior when interference avoidance was directly applied and some art was required to achieve reasonable fixed points. An understanding of the multiple receiver problem is, therefore, paramount in determining the utility of interference avoidance in real systems.

Nevertheless, owing to the simplicity of the concept and the ever increasing sophistication of radio hardware, we expect that interference avoidance will afford an interesting new perspective which might even evolve into a practical method for wireless system design. If so, we expect it to be especially useful in unlicensed bands such as the U-NII, where users can mutually interfere with officially sanctioned impunity [11].

\section{REFERENCES}

[1] "Special issue on software radio," IEEE Personal Commun. Mag., vol. 6, no. 4, Aug. 1999.

[2] I. Seskar and N. Mandayam, "Software Defined Radio Architectures for Interference Cancellation in DS-CDMA Systems," IEEE Pers. Commun. Mag., vol. 6, pp. 26-34, Aug. 1999.

[3] I. Seskar and N. B. Mandayam, "A Software Radio Architecture for Linear Multiuser Detection," IEEE J. Select. Areas Commun., vol. 17, pp. 814-823, May 1999.

[4] J. Mitola, "The software radio architecture," IEEE Commun. Mag., vol. 38, p. 26, May 1995.

[5] S. Sheng, R. Allmon, L. Lynn, I. O'Donnell, K. Stone, and R. Brodersen, "A Monolithic CMOS Radio System for Wideband CDMA Communications," in Proc. Infopad FTP Archive, 1998.

[6] S. V. Hanly, "An algorithm for combined cell-site selection and power control to maximize cellular spread spectrum capacity," IEEE J. Select. Areas Commun., vol. 13, pp. 1332-1340, Sept. 1995.

[7] R. Yates, "A framework for uplink power control in cellular radio systems," IEEE J. Select. Areas Commun., vol. 13, pp. 1341-1348, Sept. 1995.

[8] S. Ulukus and R. Yates, "Stochastic power control for cellular radio systems," IEEE Trans. Commun., vol. 46, pp. 784-798, June 1998. 
[9] S. Ulukus, "Power Control, Multiuser Detection and Interference Avoidance in CDMA Systems," Ph.D. dissertation, Rutgers University, Dept. Elect. Comp. Eng., NJ, 1998.

[10] P. Viswanath, V. Anantharam, and D. Tse, "Optimal sequences, power control and capacity of spread-spectrum systems with multiuser receivers," IEEE Trans. Inform. Theory, vol. 45, pp. 1968-1983, 1999.

[11] "FCC Report and Order 97-5: Amendment of the commission's rules to provide for operation of unlicensed NII devices in the $5 \mathrm{GHz}$ frequency range," Federal Communications Commission, ET Docket-102, 1997.

[12] H. L. Van Trees, Detection, Estimation, and Modulation Theory. New York: Wiley, 1968, pt. I.

[13] S. Verdu, Multiuser Detection. Cambridge, U.K.: Cambridge Univ. Press, 1998.

[14] A. Papoulis, Probability, Random Variables, and Stochastic Processes, 3rd ed. New York: McGraw-Hill, 1991.

[15] T. M. Cover and J. A. Thomas, Elements of Information Theory. New York: Wiley, 1991.

[16] J. M. Wozencraft and I. M. Jacobs, Principles of Communication Engineering. New York: Wiley, 1965.

[17] G. Strang, Linear Algebra and Its Applications, 2nd ed. New York: Academic, 1992.

[18] S. Haykin, Communication Systems. New York: Wiley, 1994.

[19] U. Madhow and M. L. Honig, "MMSE interference suppression for direct-sequence spread-spectrum CDMA," IEEE Trans. Commun., vol 42, pp. 3178-3188, Dec. 1994.

[20] S. Verdú, "Capacity region of Gaussian CDMA channels: The symbolsynchronous case," in Proc. Allerton Conf. Communications, Control and Computing, Oct. 1986, pp. 1025-1034.

[21] M. Rupf and J. L. Massey, "Optimum sequence multisets for synchronous code-division multiple-access channels," IEEE Trans. Inform. Theory, vol. IT-40, pp. 1261-1266, July 1994.

[22] Z. Xie, R. T. Short, and C. K. Rushforth, "A family of suboptimum detectors for coherent multiuser communications," IEEE J. Select. Areas Commun., vol. 8, pp. 683-690, May 1990.

[23] L. R. Welch, "Lower bounds on the maximum cross correlation of signals," IEEE Trans. Inform. Theory, vol. IT-20, pp. 397-399, May 1974.

[24] J. L. Massey, "On welch's bound for the correlation of a sequence set," in Proc. IEEE Int. Symp. Information Theory (ISIT'91), 1991, p. 385.

[25] J. L. Massey and T. Mittelholzer, "Welch's bound and sequence sets for code-division multiple access systems," in Sequences II: Methods in Communication, Security and Computer Science, R. Capocelli, A. De Santis, and U. Vaccaro, Eds. New York: Springer-Verlag, 1991

[26] P. Viswanath and V. Anantharam, "Optimal sequences and sum capacity of synchronous cdma systems," IEEE Trans. Inform. Theory, vol. 45, pp. 1984-1991, Sept. 1999.

[27] A. W. Marshall and I. Olkin, Inequalities: Theory of Majorization and its Applications. New York: Academic, 1979

[28] C. Rose, "Sum capacity and interference avoidance: convergence via class warfare," in Proc. CISS 2000, Princeton, NJ, Mar. 2000.

[29] _ "Cdma codeword optimization: interference avoidance and convergence via class warfare," IEEE Trans. Inform. Theory, vol. 47, pp. 2368-2382, Sept. 2001.

[30] P. B. Rapajic and B. S. Vucetic, "Linear adaptive transmitter-receiver structures for asynchronous CDMA," European Trans. Telecomm., vol. 6, pp. 21-28, Jan. 1995.

[31] M. Honig and V. Veerakachen, "Performance variability of linear multiuser detection for DS-CDMA," in Proc. IEEE Vehicular Technology Conf. VTC96, Apr. 1996, pp. 372-376.

[32] S. Ulukus and R. D. Yates, "Iterative construction of optimum signature sequence sets in synchronous CDMA systems," IEEE Trans. Inform. Theory, vol. 47, pp. 1989-1998, July 2001.

[33] — - "Iterative signature adaptation for capacity maximization of cdma systems," in Proc. Allerton Conf. Communication, Control, and Computing, Sept. 1998.

[34] P. Anigstein and V. Anantharam, "On the Convergence of the MMSE Algorithm for Interference Avoidance," in Proc. 38th Allerton Conf. Communication, Control, and Computing, Oct. 2000.

[35] M. Honig, U. Madhow, and S. Verdú, "Blind adaptive multiuser detection," IEEE Trans. Inform. Theory, vol. 41, pp. 944-960, July 1995.

[36] P. Viswanath and V. Anantharam, "Total Capacity of Vector Channels," College of Engineering, U.C. Berkeley, UCB/ERL Memorandum 99/47, 1999.

[37] F. B. Hildebrand, Advanced Calculus for Applications. Englewood Cliffs, NJ: Prentice-Hall, 1976.
[38] G. L. Nemhauser, A. H. G. Rinnooy Kan, and M. J. Todd, Eds., Optimization. North-Holland: Elsevier, 1989.

[39] D. P. Bertsekas, Constrained Optimization and Lagrange Multiplier Methods. San Diego, CA: Academic, 1982.

[40] D. Tse and S. Hanly, "Linear Multiuser Receivers: Effective Interference, Effective Bandwidth and User Capacity," IEEE Trans. Inform. Theory, vol. 45, pp. 641-657, Mar. 1999.

[41] D. C. Popescu and C. Rose, "Interference Avoidance and Dispersive Channels. A New Look at Multicarrier Modulation," in Proc. 37th Allerton Conf. Communication, Control, and Computing, Monticello, IL, Sept. 1999, pp. 505-514.

[42] _ "Fading Channels and Interference Avoidance," in Proc. 39th Allerton Conf. Communication, Control, and Computing, Monticello, IL, Sept. 2001, pp. 1073-1074.

[43] — "CDMA Codeword Optimization for Uplink Dispersive Channels Through Interference Avoidance," IEEE Trans. Inform. Theory, submitted for publication.

[44] J. Concha and S. Ulukus, "Optimization of CDMA signature sequences in multipath channels," in Proc. IEEE Vehicular Technology Conf., vol. 3, May 2001, pp. 1978-1982.

[45] S. Ulukus and R. D. Yates, "Optimum signature sequence sets for asynchronous CDMA systems," in Proc. 38th Annual Allerton Conf. Communications, Control, and Computing, Oct. 2000.

[46] — "Signature sequence optimization in asynchronous CDMA systems," in Proc. IEEE Int. Conf. Communications, vol. 2, June 2001, pp. $545-549$.

[47] — "User capacity of asynchronous cdma systems with optimum signature sequences," IEEE Trans. Inform. Theory, to be published.

[48] D. C. Popescu and C. Rose, "A New Approach to Multiple Antenna Systems: Sum Capacity Optimization Through Interference Avoidance," IEEE J. Select. Areas Commun., submitted for publication.

[49] D. Popescu and C. Rose, "Interference avoidance and multiaccess vector channels," in Proc. Conf. Int. Symp. Information Theory, Lausanne, Switzerland, July 2002.

[50] W. Yu, W. Rhee, S. Boyd, and J. M. Cioffi, "Iterative Water-Filling for Gaussian Vector Multiple Access Channels," in Proc. 2001 Int. Symp. Information Theory, Washington, DC, June 2001.

[51] D. C. Popescu and C. Rose, "Codeword Quantization for Interference Avoidance," in Proc. 2000 Int. Conf. Acoustics, Speech, Signal Processing - ICASSP 2000, vol. 6, Istanbul, June 2000, pp. 3670-3673.

[52] D. E. Tabora, "An Analysis of Covariance Estimation, Codeword Feedback, and Multiple Base Performance of Interference Avoidance,' Master's thesis, Rutgers University, Dept. Elect. Comp. Eng., NJ, 2001

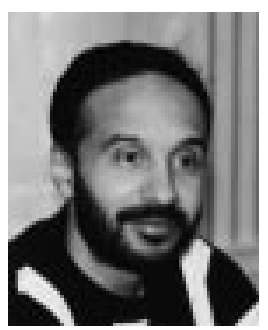

Christopher Rose (S'78-M'86) received the B.S., M.S. and Ph.D. degrees from the Massachusetts Institute of Technology, Cambridge, Massachusetts, in 1979, 1981 and 1985, respectively.

Following graduate school, he joined AT\&T Bell Laboratories, Holmdel, NJ, as a Member of the Network Systems Research Department. He is currently an Associate Professor of Electrical and Computer Engineering at Rutgers University, NJ, an Associate Director of the Wireless Networks Laboratory (WINLAB), as well as a Henry Rutgers Research Fellow. He is an editor for the ACM Wireless Networks (WINET) Journal, the Elsevier Computer Networks Journal, and has served on many conference technical program committees and was technical program Co-chair for MobiCom'97, Co-chair of the WINLAB Focus'98 on the U-NII, the WINLAB Berkeley Focus'99 on Radio Networks for Everything, and the Berkeley WINLAB Focus 2000 on Picoradio Networks. He has also served as General Chair of ACM SIGMobile MobiCom 2001 (Rome, July 2001). In December 1999, he served on an international panel to evaluate engineering teaching and research in Portugal. Closer to home, he has served on the Scientific Fields Advisory Committee of the New Jersey Commission on Science and Technology. His current technical interests include mobility management, novel mobile communications networks, applications of genetic algorithms to control problems in communications networks and most recently, interference avoidance methods using universal radios to foster peaceful coexistence in what will be the wireless ecology of the recently allocated $5 \mathrm{GHz}$ U-NII band.

Dr. Rose is currently a member of the ACM MobiCom Steering Committee and is a past member of the ACM SIGMobile Executive Committee. 


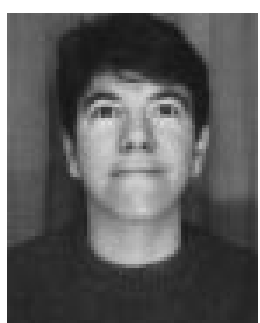

Sennur Ulukus (S'90-M'98) received the B.S. and M.S. degrees in electrical and electronics engineering from Bilkent University, Ankara, Turkey, in 1991 and 1993, respectively, and the $\mathrm{Ph} . \mathrm{D}$. degree in electrical and computer engineering from Rutgers University, NJ, in 1998.

During her Ph.D. studies, she was with the Wireless Information Network Laboratory (WINLAB), Rutgers University. From 1998 to 2001, she was a Senior Technical Staff Member at AT\&T Labs-Research, City, NJ. Since August 2001, she is an Assistant Professor in the Department of Electrical and Computer Engineering at University of Maryland, College Park, Maryland, where she also holds a joint appoinment with the Institute for Systems Research. Her research interests are in the general area of wireless communication theory, with an emphasis on power control, multiuser detection, array signal processing, and signature sequence design techniques for wireless CDMA systems.

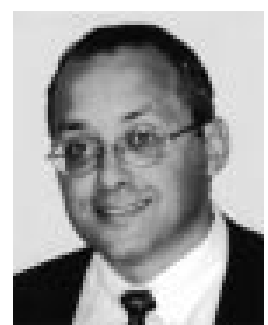

Roy D. Yates (M'91) received the B.S.E. degree in 1983 from Princeton University, Princeton, NJ, and the S.M. and Ph.D. degrees from Massachusetts institute of Technology, Cambridge, MA, in 1986 and 1990, respectively, all in electrical engineering,

Since 1990, he has been with the Wireless Information Networks Laboratory (WINLAB), Piscataway, NJ, and the Electrical and Computer Engineering Department at Rutgers University. Presently, he serves as Director of WINLAB and Associate Professor in the Electrical and Computer Engineering Department. He is an Associate Editor of the IEEE JOURNAL ON Selected AREAS IN Communications Series in Wireless Communication. He is a coauthor (with David Goodman) of "Probability and Stochastic Processes: A Friendly Introduction for Electrical and Computer Engineers" (New York: Wiley). His research interests include power control, interference suppression, and media access protocols for wireless communications systems. 